\title{
INTERSTELLAR AND INNER SOURCE PICKUP IONS OBSERVED WITH SWICS ON ULYSSES
}

\author{
GEORGE GLOECKLER \\ Department of Physics and IPST, University of Maryland, College Park, MD 20742, U.S.A. and \\ Department of Atmospheric, Oceanic and Space Sciences, University of Michigan, Ann Arbor, \\ MI 48109, U.S.A. \\ JOHANNES GEISS \\ International Space Science Institute, Hallerstrasse 6, CH-3012 Bern, Switzerland
}

\begin{abstract}
Many species of pickup ions, both of interstellar origin and from an inner, distributed source have been discovered using data from the Solar Wind Ion Composition Spectrometer (SWICS) on Ulysses. Velocity distribution functions of these ions were measured for the first time over heliocentric distances between 1.35 and 5.4 AU, both at high and low latitudes, and in the disturbed slow solar wind as well as the steady fast wind of the polar coronal holes. This has given us the first glance at plasma properties of suprathermal ions in various solar wind flows, and is enabling us to study the chemical and, in the case of He, the isotopic composition of the local interstellar cloud. Among the new findings are (a) the surprisingly weak pitch-angle scattering of low rigidity, suprathermal ions leading to strongly anisotropic velocity distributions in radial magnetic fields, (b) the efficient injection and consequent acceleration of pickup ions, especially $\mathrm{He}^{+}$and $\mathrm{H}^{+}$, in the turbulent solar wind, and (c) the discovery of a new extended source releasing carbon, oxygen, nitrogen and possibly other atoms and molecules in the inner solar system. Pickup ion measurements are now used to study the characteristics of the local interstellar cloud (LIC) and, in particular, to determine accurately the abundance of atomic $\mathrm{H}, \mathrm{He}, \mathrm{N}, \mathrm{O}$, and $\mathrm{Ne}$, the isotopes of $\mathrm{He}$ and $\mathrm{Ne}$, as well as the ionization fractions of $\mathrm{H}$ and $\mathrm{He}$ in the LIC. Pickup ion observations allow us to infer the location of the termination shock and, in combination with measurements of anomalous cosmic rays, to investigate termination shock acceleration mechanisms.
\end{abstract}

\section{Introduction}

Ions in the expanding corona are virtually all of solar origin. With increasing distance from the Sun, however, non-solar ions are gradually mixed in, so that when the solar wind approaches the termination shock (estimated to be between $\sim 85 \mathrm{AU}$ and $110 \mathrm{AU}$ in the direction of motion of the Sun through the interstellar cloud, the solar apex), their relative abundance is estimated to have reached the order of $10 \%$. These non-solar ions originate from extended sources such as the interstellar gas, interstellar grains and interplanetary grains, as well as from local sources such as comets or planetary atmospheres as is illustrated in Figure 1.

At relatively unperturbed solar wind conditions, two distinct types of ion velocity distributions are observed in interplanetary space. (1) The dominant solar wind ions of solar origin are found in a characteristically narrow Mach angle that cor- 


\section{Heliospheric Pickup Ions}

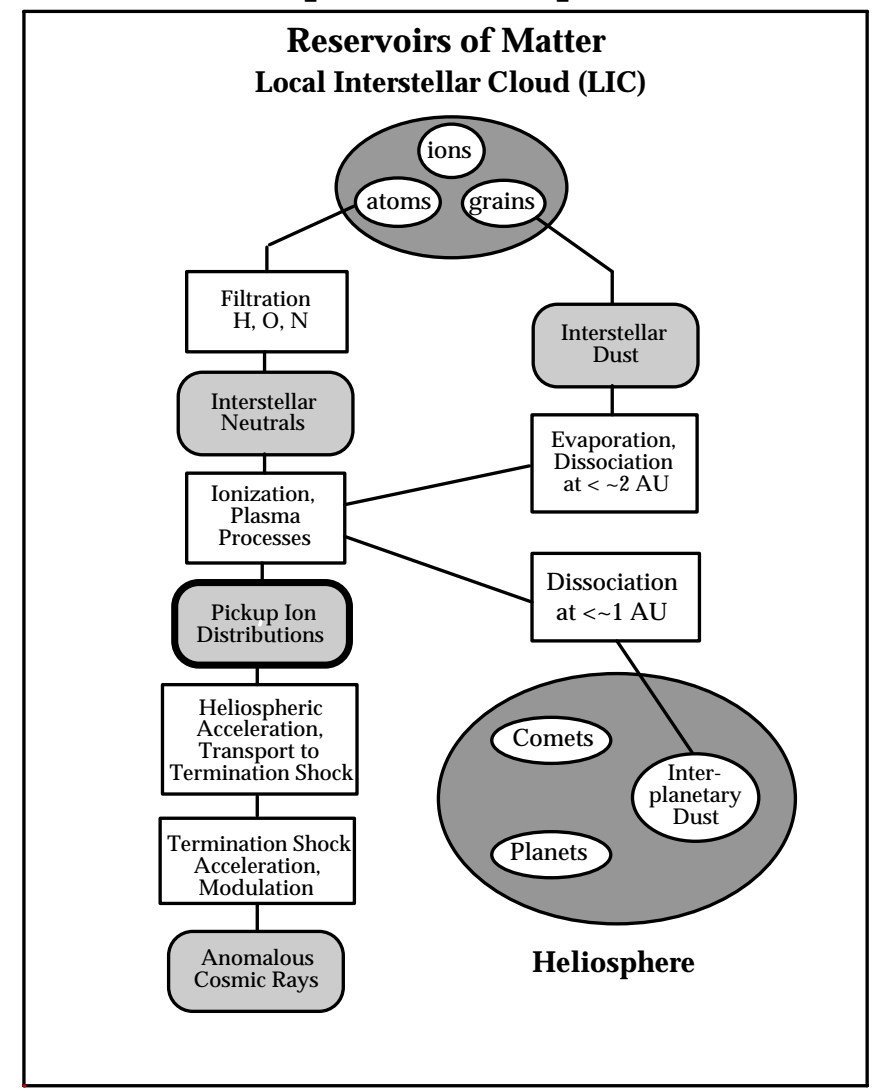

Figure 1. Schematic representation of sources and processes leading to the creation of pickup ions in the heliosphere and their subsequent transport and acceleration to form Anomalous Cosmic Rays. In addition to the interstellar source, a new extended inner source (interstellar and interplanetary dust) inside the heliosphere has been identified using SWICS/Ulysses data. The large shaded ovals represent source regions of matter shown in small ovals. Physical processes are shown in clear boxes. These processes produce the particle populations shown in the lightly shaded boxes.

responds to Mach numbers of typically 10 to 20. (2) Less abundant are the pickup ions which have broad suprathermal distribution functions that in the undisturbed solar wind show a sharp decrease at the upper velocity limit of twice the solar wind speed (Möbius et al., 1985; Gloeckler et al., 1993). At heliocentric distances of several AU and sufficiently far from local sources, their number densities are of the order of $10^{-3}$ relative to the number densities of the solar wind ions. All pickup ions that have been identified up to now are of non-solar origin.

For presenting and comparing different pickup ion populations, it is advantageous to relate $V_{\text {ion }}$, the speed of an incoming ion to $V_{\mathrm{sw}}$, the solar wind bulk speed at that time. We will use 


$$
W=V_{\text {ion }} / V_{\mathrm{sw}},
$$

for the relative speed of an ion in the reference frame of the Sun (and the spacecraft). Then

$$
\mathbf{w}=\left(\mathbf{V}_{\text {ion }}-\mathbf{V}_{\mathrm{sw}}\right) / V_{\mathrm{sw}},
$$

relates the relative velocity, $\mathbf{w}$, in the reference frame of the solar wind to the ion velocity, $\mathbf{V}_{\text {ion }}$, in the spacecraft frame.

Three criteria are available for identifying pickup ion populations and determining their sources of origin:

(1) Charge states. Solar wind ions of heavy elements have high charges reflecting the typical coronal temperature of $\sim 10^{6} \mathrm{~K}$. Pickup ions, on the other hand, are predominately singly charged. The reason for this is simple. Pickup ions, created as singly charged, are rapidly convected by the solar wind from regions of high EUV and solar wind flux that caused their ionization to distances where further ionization becomes negligible. Another way of stating this is that the time it takes to ionize an atom (by combination of photoionization by solar EUV, charge exchange with solar wind ions, and ionization by suprathermal electrons) is long compared to the time it takes to convect the pickup ion from regions of high to low ionization, a distance of several AU. The only important exception is He which also has a relatively large cross section for double ionization. Therefore, the vast majority of pickup ions produced from atoms or molecules outside magnetospheres remains singly charged. Inside magnetospheres, where ion residence lifetimes are long and electron energies, and hence ionization rates, are high, ions produced from neutral atmospheres may carry two or more charges (Young et al., 1977; Gloeckler et al., 1985; McNutt et al., 1981; Geiss et al., 1992), and thus, multiply charged ions of planetary origin may be found in the wake of planets. Measurements of ionization states of heavy ions is one of the easiest ways to separate and identify low-charge state, heavy pickup ions from the far more abundant, highly charged solar wind ions.

(2) Spatial distributions in the heliosphere. Ions produced from the interstellar gas have a very characteristic spatial distribution in the heliosphere. Their number density depends on both the angle of the observer with respect to the direction of the solar apex, as well as on the ionization rate which determines the distance from the Sun of their maximum abundance (e.g., Thomas, 1978; Holzer, 1989). The density of ions of local origin (e.g., comets, Jupiter, etc.), on the other hand, decrease with distance from the source (cf., Neugebauer et al., 1987; Geiss et al., 1994b; Ogilvie et al., 1995). However, local sources of neutrals, located directly between the Sun and the point of observation, can be detected even at large distances from the source as was recently demonstrated by Grünwaldt et al. (1997) who, with an 
instrument near Earth, recorded pickup ions produced from neutrals originating from Venus.

(3) Velocity distributions. In the solar wind flowing through the heliosphere, pickup ions are produced with $W \ll 1$ from slowly moving atoms or molecules, such as the interstellar gas atoms, cometary constituents or molecules, and atoms and ions liberated from grains by evaporation or sputtering. These ions are immediately picked up by the electromagnetic field of the solar wind, and rapidly convected outward. Pitchangle scattering and or variations in the magnetic field (B) directions are then required to spread the ions into shell-like distributions in $\mathbf{w}$ and, in particular, change $W$ of some of the ions from less than to greater than 1 . When the average direction of $\mathbf{B}$ is nearly perpendicular to $V_{\mathrm{sw}}$, i.e., for distances greater than about a few $\mathrm{AU}$ at low heliocentric latitude, distributions showing little anisotropy between $W<1$ and $W>1$ are expected and are indeed observed (Gloeckler et al., 1993, 1997). On the other hand, at high latitudes or very close to the Sun where the B field is oriented more radially, the velocity distribution of pickup ions is found to be highly anisotropic with little scattering into the $W>1$ phase-space hemisphere (Gloeckler et al., 1995). In either case, pickup ions are adiabatically cooled in the expanding solar wind. Thus, when moving away from the region where they are created, the shell-like distribution of these pickup ions shrinks to lower $w$ values. All these processes tend to produce a pickup ion velocity distribution in the solar wind frame that is relatively flat to $w_{c}=V_{\mathrm{sw}}$ with a sharp cutoff beyond $w_{c}$. These highly suprathermal pickup ion distributions are distinctly different from the thermal spectra of solar wind ions. For protons and ${ }^{4} \mathrm{He}^{++}$this difference in the velocity distributions provides the only means of separating pickup hydrogen and ${ }^{4} \mathrm{He}^{++}$from solar wind protons and alpha particles respectively.

Using one or more of the three criteria given above, the following pickup ions have been discovered: ${ }^{1} \mathrm{H}^{+}$(Gloeckler et al., 1993), ${ }^{4} \mathrm{He}^{+}$(Möbius et al., 1985; Gloeckler et al., 1993), ${ }^{3} \mathrm{He}^{+}$(Gloeckler and Geiss, 1996), ${ }^{4} \mathrm{He}^{++}$(Gloeckler et al., 1997), $\mathrm{N}^{+}, \mathrm{O}^{+}$, and $\mathrm{Ne}^{+}$(Geiss et al., 1994a). The dependence of their abundances on solar distance and latitude, as well as the shape of their velocity distributions show that the majority of the pickup ions of these species in the heliosphere as a whole is produced from the neutral interstellar gas as it flows through the solar system.

$\mathrm{C}^{+}$ions are also found at all solar latitudes and distances visited by Ulysses (cf., Figures 13 and 14). However, their distribution in space and their phase-space density spectra differ radically from pickup ions produced from the interstellar gas. Geiss et al. $(1995,1996)$ have shown that the majority of the $\mathrm{C}^{+}$ions as well as a fraction of $\mathrm{O}^{+}$and $\mathrm{N}^{+}$are produced by an 'inner source' that is located at solar distances below a few AU. These authors presented evidence showing that at medium and high solar latitudes, molecules sublimating from interstellar grains that have penetrated into the inner heliosphere constitute a significant component 
of this inner source. In Sections 3 and 4 we present new data and relate these new results to our earlier work, in order to further characterize the inner source.

\section{Hydrogen and Helium Pickup Ions}

The orbit of Ulysses and the low background capabilities of the SWICS instrument (Gloeckler et al., 1992) made it possible to discover pickup hydrogen (Gloeckler et al., 1993) whose existence was postulated in the early 1970s (e.g., Blum and Fahr, 1970; Axford, 1972, and references therein; Vasyliunas and Siscoe, 1976), and whose detection was anticipated with the launches of the deep space missions Pioneers 10 and 11 and Voyagers 1 and 2. The exceptionally low background of SWICS and the long time periods that Ulysses spent in the fast, steady and quiet solar wind from the polar coronal holes enabled us to identify the uncommon ${ }^{4} \mathrm{He}^{++}$ pickup ions and from these measurements deduce the abundance of He in the local interstellar cloud (Gloeckler et al., 1997). With SWICS we also discovered the extremely rare pickup ${ }^{3} \mathrm{He}^{+}$and used its measured abundance to place a new lower limit on the amount of missing matter in the universe (Gloeckler and Geiss, 1996).

\subsection{PICKUP IONS OBSERVED IN THE QUIET, HIGH-SPEED SOLAR WIND OF THE POLAR CORONAL HOLE}

\subsubsection{Protons}

Pickup hydrogen ions are distinguishable from the far more abundant solar wind protons by their distinctly different velocity distributions as displayed in Figure 2 . The proton phase-space density shown was accumulated over a 100-day period (10 April to 19 July 1994) when Ulysses was at an average heliocentric distance of 3.0 AU and at a high latitude $\left(-66^{\circ}\right)$ in the undisturbed and fast $\left(785 \mathrm{~km} \mathrm{~s}^{-1}\right)$ solar wind from the south polar coronal hole. The solar wind proton distribution around $W=1$ is narrow and well represented by a kappa function in the solar wind frame:

$$
\begin{aligned}
& f_{\mathrm{sw}}(w)=f_{0}\left[1+(w / \theta)^{2} / \kappa\right]^{-(\kappa+1)}, \\
& \theta=V_{\mathrm{th}}[1-(1.5 / \kappa)]^{1 / 2}
\end{aligned}
$$

The fit (curve labeled Solar Wind) to the solar wind peak around $W=1$ is the kappa distribution (Equation (3)) transformed to the spacecraft frame using Equation (2), and integrated over the instrument view angles (see Gloeckler, 1996, for details). Best values for the density, thermal speed, $V_{\mathrm{th}}$, and $\kappa$ were $0.30 \mathrm{~cm}^{-3}$, $32.3 \mathrm{~km} \mathrm{~s}^{-1}$ and 6.3 , respectively. We note in passing that a maxwellian distribution fails to fit the solar wind proton distribution we observe.

The speed distribution of pickup hydrogen, on the other hand, is broad and extends to $W=2$ at which point the phase-space density drops by about three orders of magnitude. The fact that the two distributions are quite distinct implies 


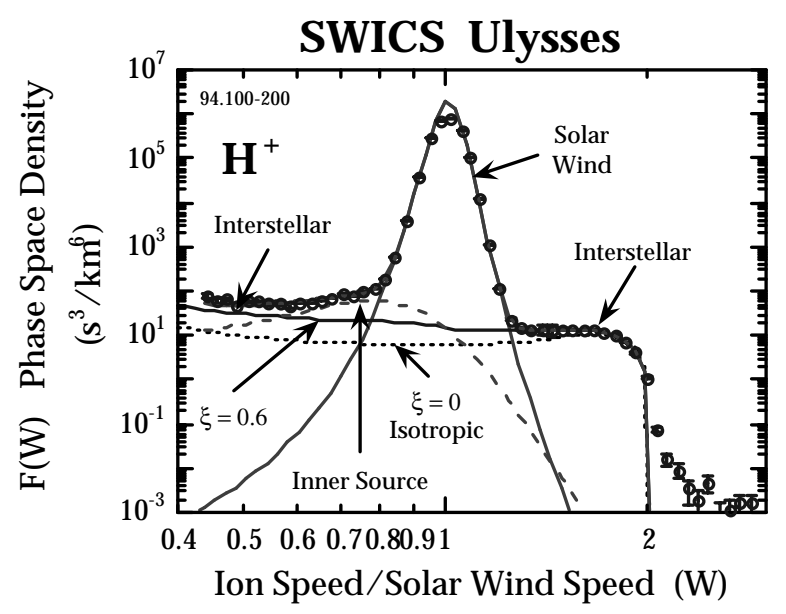

Figure 2. Phase-space density of $\mathrm{H}^{+}$versus W, the ion speed in the spacecraft frame of reference divided by the solar wind speed. This time averaged spectrum was observed with SWICS during a 100-day period in 1994 when Ulysses was in the steady high speed $\left(\sim 785 \mathrm{~km} \mathrm{~s}^{-1}\right)$ stream of the southern polar coronal hole. The average heliocentric distance and latitude were $3.0 \mathrm{AU}$ and $-66^{\circ}$ respectively, and the average direction of the magnetic field almost radial $\left(165^{\circ}\right)$. The two highest density points closest to $W=1$ fall below the kappa curve fit (labeled Solar Wind) because instrument deadtime saturated the proton count rate used here. We note that the solar wind proton number density is computed from another rate (start detector rate) which does not suffer from these deadtime effects. In these and subsequent plots the phase-space density, $F_{m, m / q}(W)$, is computed from the differential energy per charge flux of the ion with mass $(m)$ and mass/charge $(m / q)$ in the standard fashion using a calculated value for the isotropic geometrical factor. Model distribution functions are then computed, first in the solar-wind frame of reference and then transformed to the spacecraft frame, integrating over the SWICS view angles using the measured directional geometrical factor to obtain the predicted count rate for each of the $64 \mathrm{~W}$ intervals. These count rates are then converted to a predicted phase-space density for each $W$ step using the calculated isotropic geometrical factor of the instrument. (See Gloeckler, 1996 for further details.)

that the interaction between the solar wind and pickup ions is sufficiently weak so that no indication of thermalization of the pickup ion populations is apparent. The very sharp drop in phase-space density at $W=2$ indicates little energy diffusion, at least in the quiet solar wind.

The dotted curve labeled $\xi=0$ represents the predicted phase-space density of pickup protons using the 'hot model' of Thomas (1978) for the spatial distribution of hydrogen atoms in the heliosphere. We use the Vasyliunas and Siscoe (1976) equation, $f_{\text {iso }}(R, \Theta, w)$, derived under the assumption of rapid pitchangle scattering and hence isotropy (see also Gloeckler, 1996) for computing the phase-space density in the solar wind frame of reference of the resulting pickup protons at the location $(R, \Theta)$ of Ulysses, with $R$ the heliocentric radial distance in $\mathrm{AU}$ and $\Theta$ the angle between the direction of motion of the Sun relative to the interstellar cloud and the Sun-Ulysses line. Finally, we make the coordinate transformation to the spacecraft frame and integrate over the view directions of SWICS. The parameter 
values used for the density, temperature and flow speed of atomic hydrogen at the termination shock were $0.115 \mathrm{~cm}^{-3}$ (Gloeckler et al., 1997), $7000 \mathrm{~K}$, and $20 \mathrm{~km} \mathrm{~s}^{-1}$ (Lallement, 1996; Geiss and Witte, 1996) respectively. The loss rate $\left(\beta_{\text {Hloss }}=5.5 \times 10^{-7} \mathrm{~s}^{-1}\right)$ and the ratio of radiation pressure to the gravitational force $(\mu)$ were taken from the best fit to the spatial distribution of the pickup $\mathrm{H}^{+}$ measured between 1.5 and 3.3 AU (see Figure 6). The total production rate of pickup protons from atomic hydrogen required to match the observations for $W$ above $\sim 1.7$ was $\beta_{\text {Hprod }}=2.25 \times 10^{-7} \mathrm{~s}^{-1}$. This value is considerably below the measured value of the production rate $\left(3.5 \times 10^{-7} \mathrm{~s}^{-1}\right)$ due to charge exchange with solar wind protons alone. Furthermore, it is clear that the model distribution, based on the assumption of rapid pitchangle scattering, fails to account for the observed spectral shape. In fact, no isotropic (in the solar wind frame) velocity distribution of any kind would fit the observed spectrum both below and above $W=1$. Following Gloeckler et al. (1995) and Fisk et al. (1997) we therefore conclude that fast pitchangle scattering leading to isotropic phase-space density distributions in the solar wind frame does not occur during the time period and solar wind conditions of these observations.

The solid curve labeled $\xi=0.6$ uses a modified form of the Vasyliunas and Siscoe (1976) equation. Instead of fast scattering at all pitchangles we assume a strong suppression of scattering at $90^{\circ}$ pitchangle, but allow rapid scattering for all pitchangles less than as well as greater than $90^{\circ}$ (Fisk et al., 1997). The modified form of the Vasyliunas and Siscoe (1976) equation we use is $f(R, \Theta, w, \theta)=$ $f_{\text {iso }}(R, \Theta, w)(1+\xi(w))$ for $\cos (\theta)<1$ and $f(R, \Theta, w, \theta)=f_{\text {iso }}(R, \Theta, w) \times(1-$ $\xi(w))$ for $\cos (\theta)>1$, where $\theta$ is the angle between the velocity, $\mathbf{w}$, of the pickup ion and the direction of the average magnetic field $\mathbf{B}$ taken to point inward. In the present case of nearly radial $\left(173^{\circ}\right)$ average magnetic field, pickup ions created at $W \ll 1$ stay at speeds below $W=1$ with only a small fraction scattering past $90^{\circ}$ into the speed range above $W=1$. To approximate the effects of spatial transport of the pickup ion population, we assume that $\xi(w)=\xi \exp \left(-\left(1-w^{3 / 2}\right) R \phi / V_{\mathrm{sw}}\right)$ and treat $\xi$ and $\phi$ as free parameters. The degree of anisotropy, $\xi$, related to the probability of scattering through $90^{\circ}$, is defined in terms of the ratio, $\rho$, of the density in the $<90^{\circ}(W<1)$ to that in the $>90^{\circ}$ pitchangle $(W>1)$ hemisphere of phase-space, $\rho=(1+\xi) /(1-\xi)$.

The $\xi=0.6$ model distribution fits the observed pickup proton speed spectrum above $W=1.25$ quite well. The parameters used to compute this model distribution are the same as those for the $\xi=0$ model curve, except for $\beta_{\text {Hprod }}$, the total production rate and $\mu$. To obtain the best match to the observed distribution above $W=1.25$ required $\mu=1.17$ and $\beta_{\text {Hprod }}=4.95 \times 10^{-7} \mathrm{~s}^{-1}$. The contributions to the total production rate are primarily from charge exchange with solar wind protons (measured to be $3.5 \times 10^{-7} \mathrm{~s}^{-1}$ ) with the rest coming from ionization by solar UV and electron impact (Rucinski et al., 1996). For the anisotropy parameters we chose $\xi=0.6$ and $\phi=800\left(\mathrm{~km} \mathrm{~s}^{-1}\right) \mathrm{AU}^{-1}$. Using Equation (2) of Fisk et al. (1997) an anisotropy $\xi=0.6$ indicates a large $(\sim 1.2 \mathrm{AU})$ mean free path. 


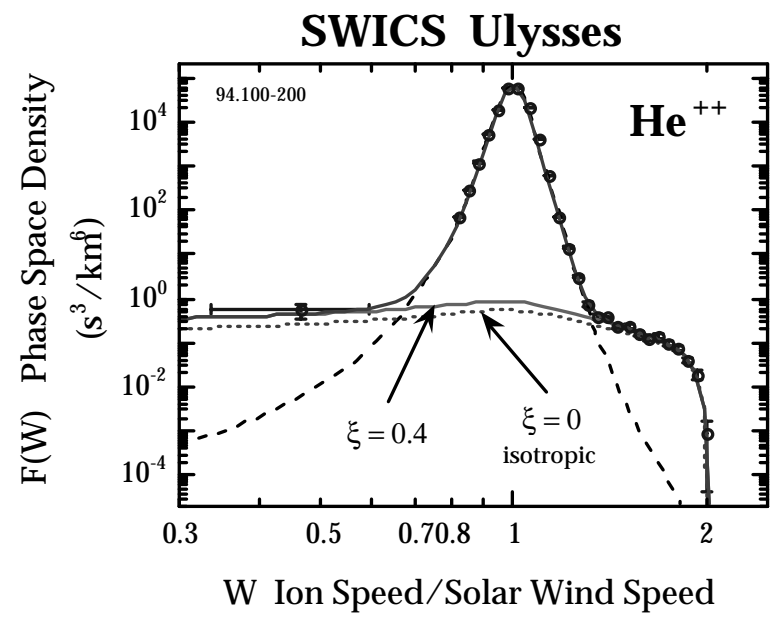

Figure 3. Same as Figure 2 for $\mathrm{He}^{++}$. This time averaged spectrum was taken during the same time period as the proton distribution of Figure 2. Pickup $\mathrm{He}^{++}$phase-space densities were used to derive the absolute interstellar helium number density of $0.0153 \mathrm{~cm}^{-3}$ because its production rate (almost entirely by double charge exchange with solar wind alpha particles) was measured by SWICS. This method eliminates instrumental systematic error because the interstellar He density is then essentially proportional to the ratio of the pickup $\mathrm{He}^{++}$flux (at $W$ close to 2 ) and the solar wind $\mathrm{He}^{++}$flux (at $W=1$ ). Because of the lower count rate no saturation of the rate near $W=1$ occurs.

While the $\xi=0.6$ fit matches the observed spectrum above $W=1.25$ extremely well it falls below the measured distribution for $W<0.85$. When data from the $45^{\circ}$ Sun sector is excluded (as was done in Gloeckler et al., 1995) the observed phase-space density below $W \sim 0.62$ falls right on the $\xi=0.6$ curve. To account for the relatively large contributions to the total density below $W=0.8$, especially in the Sun sector, we postulate the existence of an inner source of pickup protons as indicated by the dashed curve. The shape of this 'inner source' proton spectrum is similar to that of the inner source carbon and oxygen distribution discussed in Section 3 and shown in Figures 11 and 13 below.

\subsubsection{Doubly Charged Helium-4}

In Figure 3 we show the ${ }^{4} \mathrm{He}^{++}$speed distribution averaged over the same 100day period in 1994 used for protons of Figure 2 above. The distribution of alpha particles is very similar to that of $\mathrm{H}^{+}$, showing the clear separation of the solar wind alphas from interstellar pickup ${ }^{4} \mathrm{He}^{++}$, which is produced almost entirely by double charge exchange of atomic helium with solar wind alpha particles (Gloeckler et al., 1997; Gloeckler, 1996; Rucinski et al., 1998). The distribution function of pickup ${ }^{4} \mathrm{He}^{++}$(solid curve) is again anisotropic with $\phi=800\left(\mathrm{~km} \mathrm{~s}^{-1}\right) \mathrm{AU}^{-1}$ and $\xi \approx 0.4$, which corresponds to a mean free path of $\sim 1$ AU using Equation (2) of Fisk et al. (1997). To obtain the model pickup ${ }^{4} \mathrm{He}^{++}$distribution function we used the hot model of Thomas (1978) with a neutral interstellar helium density of $0.0153 \mathrm{~cm}^{-3}$ 


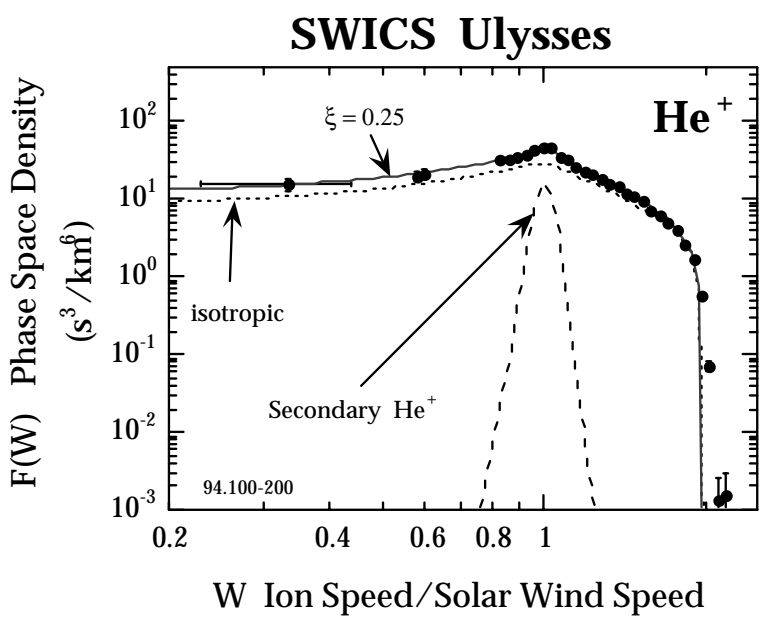

Figure 4. Same as Figure 3 for $\mathrm{He}^{+}$. Although pickup $\mathrm{He}^{+}$is about 30 times more abundant than $\mathrm{He}^{++}$, it could not be used to compute the interstellar helium density because its rate of production, almost entirely by solar UV, was not measured during the time of these observations. See text for further details and explanation of the various model curves.

(Gloeckler et al., 1997), a temperature of $7000 \mathrm{~K}$ (Witte et al., 1993, 1996) and a loss rate of $0.55 \times 10^{-7} \mathrm{~s}^{-1}$ (see Figure 4; Rucinski et al., 1996). Ninety percent of total production rate comes from double charge exchange of solar wind alphas with interstellar atomic $\mathrm{He}$ and the rest from charge exchange with pickup ${ }^{4} \mathrm{He}^{+}$ and photoionization (Gloeckler et al., 1997). Thus, the dominant portion of the total production rate of $0.0215 \times 10^{-7} \mathrm{~s}^{-1}$ used here is known reasonably well from the product of the measured solar wind alpha particle flux (adjusted to $1 \mathrm{AU}$ ) and the double charge exchange cross section of $1.9 \times 10^{-16} \mathrm{~cm}^{2}$ (Rucinski et al., 1998) at an energy corresponding to the $\sim 800 \mathrm{~km} \mathrm{~s}^{-1}$ wind. Best values for the solar wind alpha particle density, thermal speed, $V_{\mathrm{th}}$ and $\kappa$ were $0.0145 \mathrm{~cm}^{-3}, 36 \mathrm{~km} \mathrm{~s}^{-1}$, and 5.2 , respectively.

\subsubsection{Singly Charged Helium-4}

Interstellar ${ }^{4} \mathrm{He}^{+}$pickup ions are the most abundant suprathermal ions at $1 \mathrm{AU}$ and comparable in density to pickup protons at $\sim 5 \mathrm{AU}$. They were the first of the interstellar pickup ions to be discovered (Möbius et al., 1985), although the detailed spectrum of these ions over a wide velocity range was obtained only recently with the SWICS/Ulysses instrument (Gloeckler et al., 1993). Figure 4 shows the ${ }^{4} \mathrm{He}^{+}$ phase-space density as a function of $W$, the speed of ${ }^{4} \mathrm{He}^{+}$divided by the solar wind ${ }^{4} \mathrm{He}^{++}$speed. The time period for this average spectrum was the same as used for the two previous figures. SWICS samples almost all of the relevant phasespace in the case of pickup ${ }^{4} \mathrm{He}^{+}$. We show for the first time the ${ }^{4} \mathrm{He}^{+}$phase-space density over the entire interval of speeds between $\sim 0.2<W<\sim 2$. The small gaps around $W=0.5$ and $W=0.7$ result from spillover of solar wind protons and 
alpha particles for which no corrections have as yet been made. To obtain the ${ }^{4} \mathrm{He}^{+}$ densities in the important speed interval around $W=1$ we carefully subtracted known contributions from the measured fluxes of solar wind $\mathrm{Si}^{+7}, \mathrm{Mg}^{+6}, \mathrm{Fe}^{+14}$, as well as $\mathrm{O}^{+6}$ and $\mathrm{Fe}^{+12}$. The solid and dotted curves are the predicted spectra using the same parameters as for the computation of the ${ }^{4} \mathrm{He}^{++}$pickup ion distribution, except for the production rate. The dotted curve, which assumes an isotropic distribution, does not fit the measured distribution. The solid curve, which fits the data over the entire speed range, uses an anisotropy factor $\xi=0.25$, corresponding to a mean free path of $\sim 0.8 \mathrm{AU}$, and $\phi=800\left(\mathrm{~km} \mathrm{~s}^{-1}\right) \mathrm{AU}^{-1}$. The total production rate required to match the data was $0.8 \times 10^{-7} \mathrm{~s}^{-1}$. We note the major part of the production rate of ${ }^{4} \mathrm{He}^{+}$is due to photoionization. The EUV photon flux was not measured during the present observation period.

In the region around $W \approx 1$ significant contributions to the production rate of pickup ${ }^{4} \mathrm{He}^{+}$can also come from electron impact ionization close $(<1 \mathrm{AU})$ to the Sun (e.g., Rucinski et al., 1996, 1998). In this same W interval there are also contributions from 'secondary' ${ }^{4} \mathrm{He}^{+}$, produced primarily in the charge exchange reaction $\left(\sigma=8.5 \times 10^{-16} \mathrm{~cm}^{2}\right.$, Rucinski et al., 1998) of solar wind alpha particles with atomic hydrogen. In this reaction ${ }^{4} \mathrm{He}^{+}$is produced with the same velocity distribution as solar wind ${ }^{4} \mathrm{He}^{++}$, as well as a small fraction of pickup hydrogen. For the time period used in this analysis we calculate the production rate and flux of secondary ${ }^{4} \mathrm{He}^{+}$to be $0.078 \times 10^{-7} \mathrm{~s}^{-1}$ and $300 \mathrm{~cm}^{-2} \mathrm{~s}^{-1}$, respectively using the measured solar wind alpha parameters and the atomic hydrogen spatial distribution deduced from our pickup proton measurements. The dashed curve in Figure 4 represents the calculated secondary ${ }^{4} \mathrm{He}^{+}$velocity distribution whose shape is taken to be the same as that of the measured solar wind alpha particles of Figure 3. Adding the secondary ${ }^{4} \mathrm{He}^{+}$to our model fit did not entirely account for the small peak in the ${ }^{4} \mathrm{He}^{+}$phase-space density around $W=1$. To obtain the excellent fit (solid curve) to the observed spectrum over the entire range of speeds required additional production of ${ }^{4} \mathrm{He}^{+}$by electron impact ionization. The production rate we used was $\beta_{\mathrm{el}}=\left(0.16 \times 10^{-7}\right) R^{-2.3} \mathrm{~s}^{-1}$, with $R$ the heliocentric distance in AU.

\subsubsection{Singly Charged Helium-3}

The rarest of the light-mass pickup ions observed is ${ }^{3} \mathrm{He}^{+}$. Long accumulation times were required to positively identify this uncommon pickup ion and measure its abundance relative to ${ }^{4} \mathrm{He}^{+}$. The mass/charge distribution of ions with masses between 1.75 and 4.6 amu and $1.6<W<2.0$ measured by SWICS during the 40-month period (July 1992 to November 1995) of high-speed solar wind is given in the top panel of Figure 5. The triple coincidence requirement reduced residual background to about $10 \%$ of the ${ }^{3} \mathrm{He}^{+}$counts (less than one count in three years). While limited by counting statistics, the peak due to ${ }^{3} \mathrm{He}^{+}$is clearly visible at mass per charge around 3, between the peaks at mass/charge 2 and 4 from the far more abundant pickup ${ }^{4} \mathrm{He}^{++}$and ${ }^{4} \mathrm{He}^{+}$, respectively. 

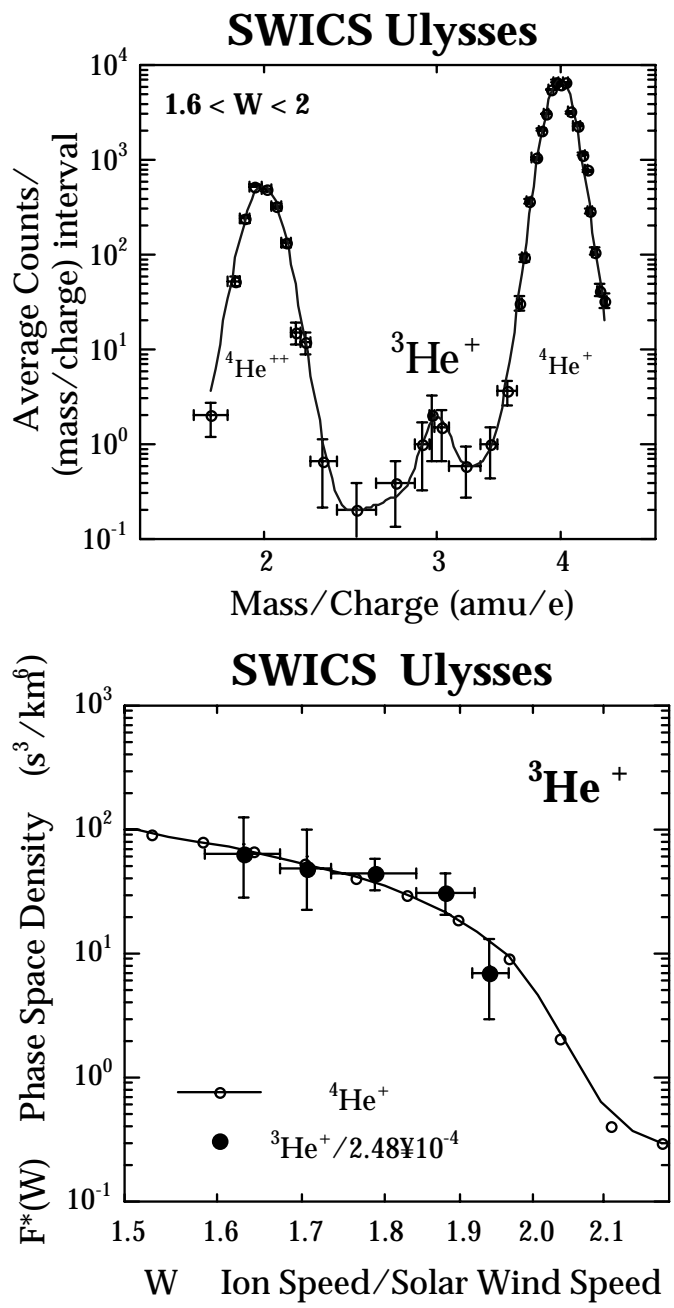

Figure 5. Top panel: plot of the average triple-coincidence counts of ions with masses between 1.75 and $4.6 \mathrm{amu}$ and with normalized speed $\mathrm{W}$ between 1.6 and 2 per mass/charge $(\mathrm{m} / q)$ interval versus the mean $m / q$ of each interval. Triple coincidence is required to determine both the mass and the mass/charge of the ions (see Gloeckler et al., 1992) and to reduce the background sufficiently to detect these extremely rare ions. In regions of low count rate the $m / q$ intervals were increased to contain at least one count. The peak at $m / q$ of $\sim 3$ is due to ${ }^{3} \mathrm{He}^{+}$. Note that ${ }^{3} \mathrm{He}^{+}$is well resolved from the far more abundant pickup ${ }^{4} \mathrm{He}^{+}$to the right, and pickup ${ }^{4} \mathrm{He}^{++}$to the left. The time interval between the detection of adjacent ${ }^{3} \mathrm{He}^{+}$followed a Poisson distribution with a mean time interval of 121 days. Because of the extremely low fluxes of ${ }^{3} \mathrm{He}^{+}$, data were taken during a 40-month period when the solar wind speed exceeded $750 \mathrm{~km} \mathrm{~s}^{-1}$ for maximum detection efficiency. Bottom panel: phase-space density of ${ }^{4} \mathrm{He}^{+}$and ${ }^{3} \mathrm{He}^{+}$(divided by $2.48 \times 10^{-4}$ ) versus $W$ (ion speed/solar wind speed). Despite the large statistical error for ${ }^{3} \mathrm{He}^{+}$, the similarity of the two spectral shapes is obvious. $\mathrm{No}^{3} \mathrm{He}^{+}$counts were detected above $W=2$ consistent with the expected cutoff in density. Below $W \sim 1.5$ the probability of ${ }^{3} \mathrm{He}^{+}$producing triple coincidence becomes extremely small since such ions have insufficient energy to trigger the $40 \mathrm{keV}$ threshold solid-state detector. 
The phase-space density of ${ }^{3} \mathrm{He}^{+}$(divided by $2.48 \times 10^{-4}$ ) is compared to that of ${ }^{4} \mathrm{He}^{+}$in the bottom panel of Figure 5. Within the statistical errors the two spectra are comparable as expected, further strengthening the case for the correct identification of interstellar ${ }^{3} \mathrm{He}$. We also note that ${ }^{3} \mathrm{He}^{+}$was not detected above $W=2$ indicating the characteristic cutoff of a pickup ion spectrum. Based on the results shown in Figure 5, Gloeckler and Geiss $(1996,1998)$ were able to place a new lower limit on the amount of missing matter in the early Universe, and concluded that the amount of ${ }^{3} \mathrm{He}$ production by stars was less than predicted by some models of stellar chemical evolution.

\subsubsection{Spatial Distribution of Atomic Hydrogen}

The spatial distribution of an atomic species from which the corresponding pickup ions are created depends on the loss rate, $\beta_{\text {loss }}$, for removing neutrals through ionization, and on $\mu$, the ratio of the force on the atom due to radiation pressure to the gravitational force. The loss rate and $\mu$ can be obtained from the shape of the distribution function of the pickup ion species between $W \sim 1.3$ and 2 , as was discussed above, or from measurements of the gradual change (gradient) of the pickup ion fluxes with heliocentric distance $R$ at a given angle $\Theta$ with respect to the direction of the solar apex. The gradient of the ratio of pickup hydrogen to pickup ${ }^{4} \mathrm{He}^{+}$is shown in Figure 6 which includes data for the time periods of Figures 2-4. Assuming $\mu=1$ for protons and $\mu=0$ for $\mathrm{He}$, the best fit to the observed gradient is with $\beta_{\text {loss }}$ (hydrogen $)=(5.5 \pm 1.0) \times 10^{-7} \mathrm{~s}^{-1}$. This same value for the loss rate was used to derive the model pickup proton distribution shown in Figure 2.

\subsection{ACCELERATED PICKUP IONS IN THE DISTURBED SOLAR WIND INSIDE A CO-ROTATING INTERACTION REGION}

Ion velocity distributions observed in the turbulent solar wind, such as the wind inside co-rotating interaction regions (CIRs) have a distinctly different character, showing unmistakable evidence for heating and acceleration. This is best illustrated for ${ }^{4} \mathrm{He}^{+}$, a pure pickup ion species. In Figure 7 we compare the phase-space density of ${ }^{4} \mathrm{He}^{+}$measured by SWICS during a $\sim 2$-day period immediately behind the reverse shock of a CIR (28 and 29 December 1992), to the distribution functions observed during a $\sim 3$-day period immediately upstream of the reverse shock of that CIR, and in the quiet polar coronal hole wind (from Figure 4), respectively. We have removed the $\left(V_{\mathrm{sw}} / V_{\mathrm{swCIR}}\right)^{4}$ dependence of the phase-space density on the solar wind speed (e.g., Vasyliunas and Siscoe, 1976) by multiplying the preshock spectrum by 2.97 and the quiet distributions by 6.24 . The difference between the CIR and quiet time spectra is most striking. While the quiet time distribution has a sharp cutoff at $W=2$ indicating negligible energy diffusion and acceleration, the CIR spectrum has a pronounced high-velocity tail showing unmistakable acceleration above $W=\sim 1.5$. We conclude, in agreement with Gloeckler et al. (1994) and Schwadron et al. (1996), that in the turbulent solar wind behind the 


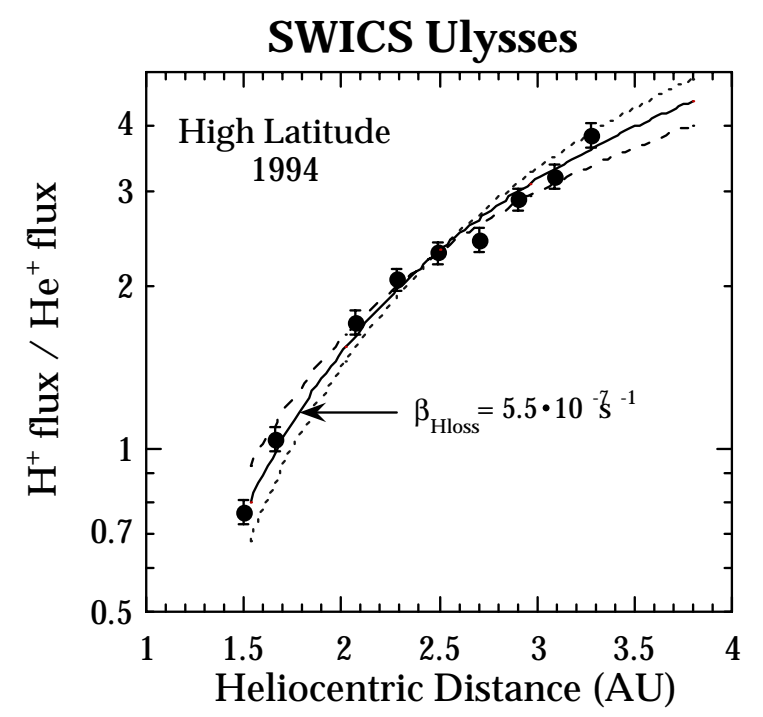

Figure 6. Ratio of $\mathrm{H}^{+}$to ${ }^{4} \mathrm{He}^{+}$pickup ion fluxes versus heliocentric range. The flux ratio is obtained from the measured phase-space densities of $\mathrm{H}^{+}$and $\mathrm{He}^{+}$for $W$ between 1.75 and 2. Each data point is a one-month average. During the entire time period of these observations Ulysses was in the high speed stream of the southern polar coronal hole. The best fit to the data is the solid curve which was computed assuming $\mu$, the ratio of radiation to gravitational forces on hydrogen to be 1 , using a hydrogen loss rate of $5.5 \times 10^{-7} \mathrm{~s}^{-1}$, a He loss rate (Rucinski et al., 1996) of $0.55 \times 10^{-7} \mathrm{~s}^{-1}$, and a $\mathrm{H} / \mathrm{He}$ density ratio at the termination shock of 7.5. The dashed and dotted curves were calculated using $\mathrm{H}$ loss rates of $4.5 \times 10^{-7} \mathrm{~s}^{-1}$ and $6.5 \times 10^{-7} \mathrm{~s}^{-1}$, respectively, but keeping the other parameters the same as before.

shock, pickup ${ }^{4} \mathrm{He}^{+}$ions are easily accelerated. Thus, the once puzzling ubiquitous presence of energetic $\left(0.4-0.6 \mathrm{MeV} \mathrm{amu}^{-1}\right){ }^{4} \mathrm{He}^{+}$measured during a $\sim 1.5$-year period in 1978-1979 at 1 AU by Hovestadt et al. (1984), can now be quite naturally explained to be interstellar pickup ${ }^{4} \mathrm{He}^{+}$that were accelerated in CIRs at $\sim 3$ to $6 \mathrm{AU}$ and then transported back to $1 \mathrm{AU}$. The distribution function immediately upstream of the reverse shock shows a modest density enhancement (tail) above the cutoff at $W=2$. This tail probably represents ions accelerated by the turbulence within the CIR that have gained enough energy to make their way back into the upstream region. It is this tail population upstream of the shock that may be further accelerated to higher energy by the shock mechanism.

In the top panel of Figure 8 we compare the spectral shapes of $\mathrm{H}^{+}, \mathrm{He}^{+}$, and $\mathrm{He}^{++}$measured downstream of the forward shock (FS) with those observed in the downstream region of the reverse shock (RS) in the late December 1992 CIR. The normalization factors applied to the reverse shock data are shown in the figure in parentheses. There are several remarkable features apparent from these data. First, we note that more $\mathrm{He}^{+}$than $\mathrm{He}^{++}$is accelerated even though solar wind alpha particles are at least a factor of $10^{3}$ more abundant than pickup $\mathrm{He}^{+}$. Second, the spectral shapes in the high speed $(W>2.4)$ tail region behind the reverse shock 


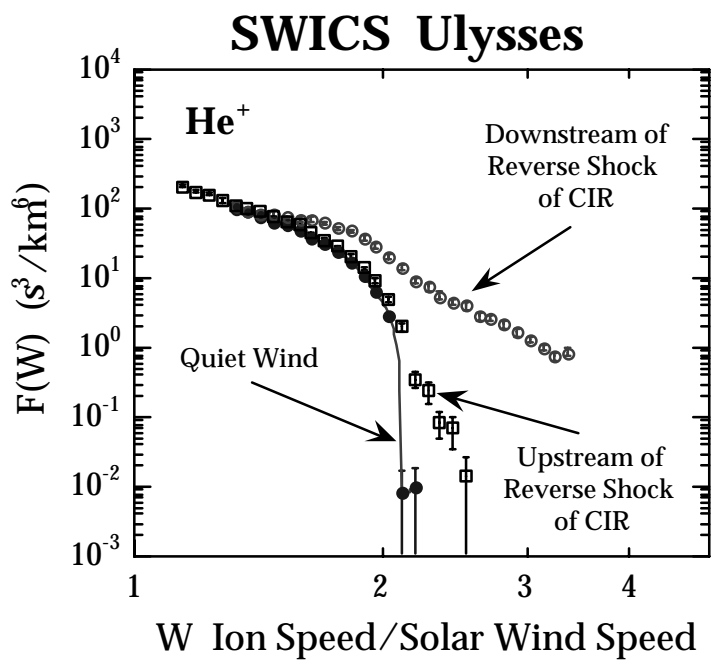

Figure 7. Phase-space density versus $W$ of ${ }^{4} \mathrm{He}^{+}$(a) during a 40-hour period at the end of 1992 (December 28, 07:47 to December 29, 23:47) behind (downstream) the reverse shock (RS) of a co-rotating interaction region (CIR), (b) during a $\sim 3$-day period immediately upstream of the same reverse shock (December 29, 23:47 to January 1, 1993, 00:59), and (c) during the extended quiet time period in the high speed solar wind (from Figure 4). Ulysses was at $-22.6^{\circ}$ and $5.06 \mathrm{AU}$ from the Sun in late December, 1992. In the region downstream of the RS the average solar wind He bulk speed was $498 \mathrm{~km} \mathrm{~s}^{-1}$, while upstream of the RS the average speed was $654 \mathrm{~km} \mathrm{~s}^{-1}$. The spectrum downstream of the reverse shock has a pronounced high-energy tail above $W \sim 1.5$ showing clear evidence of acceleration of pickup ${ }^{4} \mathrm{He}^{+}$.

are identical (within experimental uncertainties) to those behind the forward shock. This is even more remarkable because the two shocks are so different. The FS is weaker $\left(M_{s}=1.55\right)$ than the $\operatorname{RS}\left(M_{s}=2.38\right)$ and one is quasi-perpendicular while the other quasi-parallel (Balogh et al., 1995). Our observation that the acceleration process produces spectra of identical shapes despite the large difference in the shock parameters suggests that the acceleration is not due to the shocks themselves but rather to the turbulence in the downstream regions of the shocks (Gloeckler et al., 1994, see also Schwadron et al., 1996). It is evident from the solar wind phase-space densities that the solar wind behind the reverse shock is heated more than the wind downstream of the weaker forward shock. This heating supplies more suprathermal solar wind ions for injection into the acceleration process downstream of the RS than in the region behind the forward shock. The fact that four times more $\mathrm{He}^{++}$and only 1.7 times more $\mathrm{H}^{+}$and $\mathrm{He}^{+}$is accelerated behind the $\mathrm{RS}$ compared to the FS implies that a large percentage of pickup protons is accelerated in the FS. The heating of solar wind protons behind the forward shock does not provide enough suprathermal solar wind protons to completely overwhelm the pickup protons that appear as the hump in the FS spectrum (open circles).

In the bottom panel of Figure 8 we compare the phase-space density of protons (open squares) in the downstream region of the CIR reverse shock (same time 

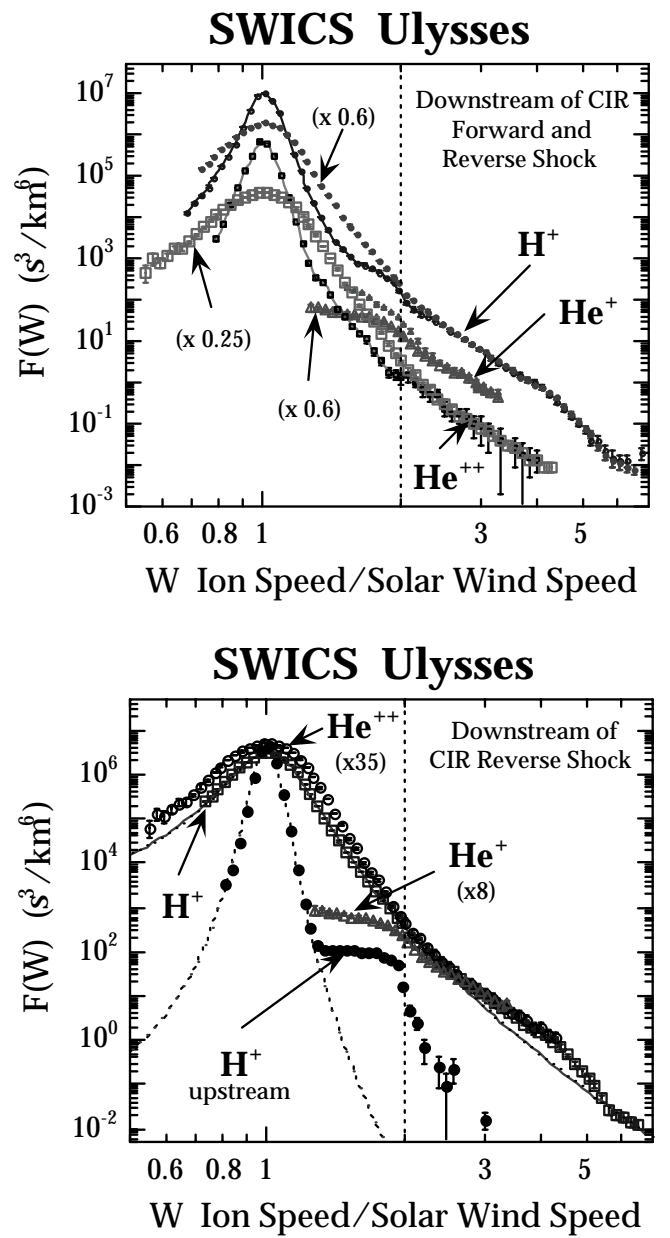

Figure 8. Top panel: distribution functions of $\mathrm{H}^{+}, \mathrm{He}^{+}$and $\mathrm{He}^{++}$downstream of the forward shock (open circles, small triangles and small squares respectively) and downstream of the reverse shock (filled circles, large triangles and large squares respectively) of the late December 1992 CIR. The time periods used for averaging the downstream FS spectra was from December 26, 19:41 to December 28, 07:47. For the downstream reverse shock (RS) distributions the time interval is the same as was used in Figure 7. The downstream RS distributions have been multiplied by the factors shown in parenthesis in order to match the respective spectra in the high velocity region above $W=2.4$. Notice the remarkable similarity in the spectral shapes of all three ion species in the high velocity tail region. Bottom panel: velocity distributions of $\mathrm{H}^{+}$(squares), $\mathrm{He}^{+}$(triangles), and $\mathrm{He}^{++}$(open circles) in the region downstream of the RS. The accumulation times were the same as those used for the distributions in Figure 7. The $\mathrm{He}^{+}$and $\mathrm{He}^{++}$phase-space densities were multiplied by 8 and 35, respectively, in order to match the proton spectrum in the high velocity tail above $W \approx 2.5$. For comparison we also show the proton distribution (solid circles) in the upstream RS region (same time period as used for the $\mathrm{He}^{+}$upstream RS spectrum of Figure 7). We note that (a) the tails (above $W \approx 2.5$ ) of all three ion species have the same shape in the downstream region of the shock, and that the shape is not a simple power law as predicted by standard shock acceleration models, (b) the $\mathrm{He}^{+} / \mathrm{He}^{++}$ratio in the tail is $\sim 4.4$, and (c) the $\mathrm{H}^{+} /\left(\mathrm{He}^{+}+\mathrm{He}^{++}\right)$ratio in the tail is about 6.5 . The upstream RS distribution shows little evidence of acceleration. 
period as in Figure 7) with that of $\mathrm{He}^{+}$(triangles) of Figure 7 (multiplied by a factor of 8 ) and $\mathrm{He}^{++}$(multiplied by 35). These normalization factors were chosen so that all three velocity distributions overlapped in the tail region above $W \sim$ 2.4. For reference we also show the distribution function of protons (filled circles) measured in the upstream region during the same time period as in Figure 7. In comparing the upstream to the downstream $\mathrm{H}^{+}$distribution we note that the solar wind proton distribution in the downstream region behind the reverse shock is very broad and non-maxwellian. The solid curve is a kappa function fit (Equation (3)) to protons with $V_{\text {th }}=67.2 \mathrm{~km} \mathrm{~s}^{-1}$ and an extremely low value of $\kappa=3.1$. This is to be contrasted with the upstream solar wind proton distribution (dotted curve) which is much colder $\left(V_{\mathrm{th}}=29 \mathrm{~km} \mathrm{~s}^{-1}\right)$ and has a weaker tail $(\kappa=4.9)$. The pickup hydrogen distribution, quite obvious in the upstream spectrum is completely obscured by the over-ten-times-more-abundant heated solar wind protons above $W \sim 1.5$ in the downstream region. We therefore conclude that, in the case of strong heating illustrated here, mostly solar wind protons are injected for further acceleration to higher energies. On the other hand, in instances where less heating occurs (as shown to be the case downstream of the FS in this CIR and as is often the case behind forward shocks of CIRs in general) the protons in the high-velocity tail will be a mixture of solar wind and pickup protons (Gloeckler et al., 1994).

The speed distributions of all three species in the tail region have identical shapes above $W \sim 2.4$. This, combined with the fact that the FS and RS spectra also have the same shapes (see left-hand panel of Figure 8), implies that the acceleration mechanism depends primarily on the ion speed. In addition, the spectral shapes are not simple power laws, as would be predicted by standard shock acceleration models, providing further evidence that the high velocity tail distributions we observe are not produced by a simple shock acceleration mechanism.

\section{Pickup Ions with Masses Heavier than Helium}

Heavy pickup ions $\left(\mathrm{O}^{+}, \mathrm{N}^{+}\right.$, and $\left.\mathrm{Ne}^{+}\right)$of interstellar origin were discovered (Geiss et al., 1994a) using SWICS/Ulysses data at distances beyond several AU. The presence of singly charged heavy ions of non-solar origin is clearly evident from Figure 9 which shows a mass/charge (m/q) histogram of ions with speeds above 1.4 times the solar wind speed $(W>1.4)$ in the mass/charge range above 10 amu $\mathrm{e}^{-1}$. The prominent peak at $m / q=16$ is due to singly charged interstellar pickup oxygen, with smaller peaks at $m / q$ of 14 and 20 corresponding respectively to pickup $\mathrm{N}^{+}$and $\mathrm{Ne}^{+}$. In particular, $\mathrm{C}^{+}(m / q=12)$ is not observed in this velocity range, placing a rough upper limit on $\mathrm{C}^{+} / \mathrm{O}^{+}$of 0.05 . These observations are consistent with an interstellar origin for these three heavy pickup ion species. Atomic O, $\mathrm{N}$, and Ne penetrate deep into the solar system before they become pickup ions. Carbon, on the other hand, is mostly ionized in the local interstellar cloud (e.g., 


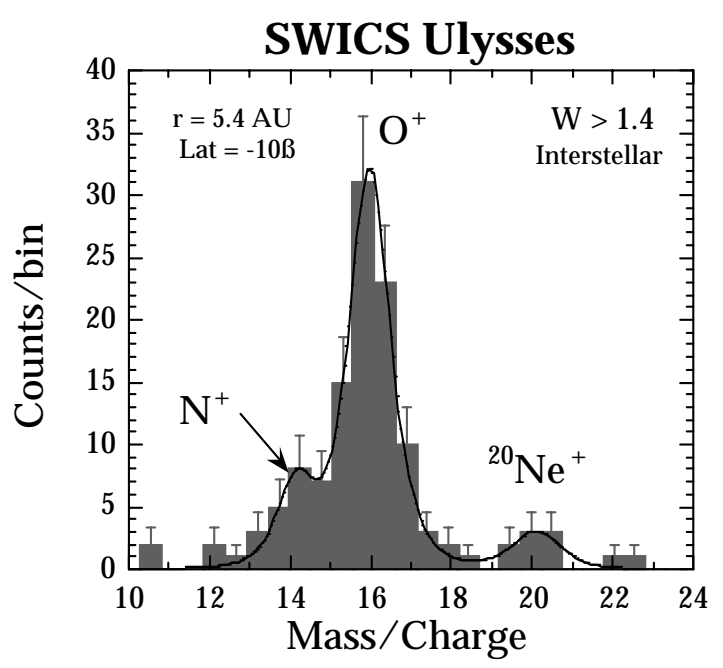

Figure 9. Plot of the double coincidence counts of ions with normalized speed $W$ above 1.4 per mass/charge $(\mathrm{m} / \mathrm{q})$ intervals versus the mean $\mathrm{m} / \mathrm{q}$ of each interval. These SWICS data were accumulated during a 135-day period in 1992 at an average distance of 5.4 AU and average helio-latitude of $-10^{\circ}$ (also see Geiss et al., 1994a). The average solar wind speed was $442 \mathrm{~km} \mathrm{~s}^{-1}$. In addition to the prominent peak at mass/charge $16\left({ }^{16} \mathrm{O}^{+}\right),{ }^{20} \mathrm{Ne}^{+}$and ${ }^{14} \mathrm{~N}^{+}$are also clearly observed. The speed $(W)$ selection criteria exclude solar wind heavy ions whose distribution peaks around $W=1$.

Frisch, this issue). Thus, a large fraction of interstellar $\mathrm{C}$ is excluded from the heliosphere.

Conclusive evidence for interstellar pickup oxygen comes from the velocity distribution function of $\mathrm{O}^{+}$shown in Figure 10. These SWICS data (and those shown in Figure 9) were accumulated during a 135-day period in 1992 at a distance of 5.4 AU and an average heliolatitude of $-10^{\circ}$. During this time period the solar wind speed remained relatively steady at $442 \pm 36 \mathrm{~km} \mathrm{~s}^{-1}$. The distribution has the characteristic cutoff at $W=2$, and the flat shape of the spectrum is similar to that of pickup hydrogen. Both the sharp cutoff and the flatness of the distribution function indicate a source location beyond 5.4 AU, the heliocentric distance of Ulysses when these data were obtained. A Jovian origin was ruled out by Geiss et al. (1994b). The smooth dotted curve is a model fit for the interstellar oxygen using $12 \times 10^{-7} \mathrm{~s}^{-1}$ for the loss and production rates of O (see Rucinski et al., 1996) and the same anisotropy factor as for hydrogen $(\xi=0.6)$. The agreement with the observed spectrum above $W=1.3$ is excellent. We find the atomic oxygen number density at the termination shock to be $7.8 \times 10^{-5} \mathrm{~cm}^{-3}$.

For $W$ between 0.8 and $\sim 1.2$ additional $\mathrm{O}^{+}$, beyond that expected from the interstellar gas source is clearly evident. This extra component of pickup $\mathrm{O}^{+}$has most likely the same origin as pickup $\mathrm{C}^{+}$discovered by Geiss et al. (1995). This hypothesis is confirmed by comparing, in Figure 11, the pickup carbon spectrum observed during this period with the pickup oxygen spectrum from which the in- 


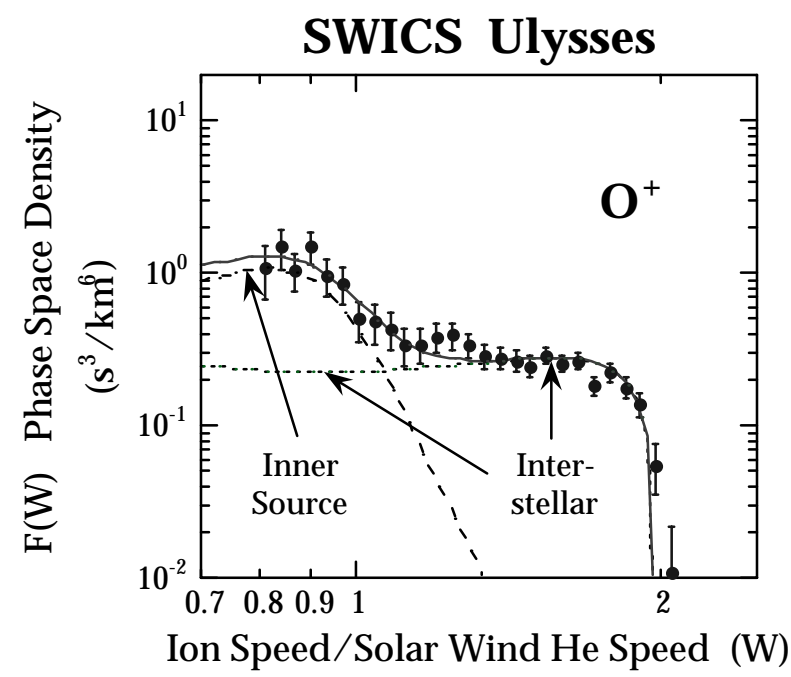

Figure 10. Phase-space density of $\mathrm{O}^{+}$versus $\mathrm{W}$ averaged over a 135 days from 17 February to 1 July 1992 when Ulysses was still near the ecliptic plane. The average solar wind speed was relatively steady at $442 \pm 36 \mathrm{~km} \mathrm{~s}^{-1}$ and the distance from the Sun remained close to $5.4 \mathrm{AU}$ during the entire time period of these observations. The dotted curve is the model calculation of interstellar pickup $\mathrm{O}^{+}$ assuming an anisotropy factor $\xi=0.6$ (see text). However, because the average magnetic field was nearly perpendicular $\left(112^{\circ}\right)$ to the solar wind flow direction the distribution function would appear to be isotropic in the spacecraft frame with nearly equal densities above and below $W=1$. While the agreement between the model and data is excellent above $W$ of about 1.3 a significant amount of $\mathrm{O}^{+}$from another source is evident below $W \approx 1.2$. A hypothetical inner source $\mathrm{O}^{+}$spectrum, represented by the dashed curve, combined with the interstellar $\mathrm{O}^{+}$curve would match the observed distribution. Below $W$ of $\sim 0.8$ significant corrections for background from low-charge-state heavy solar wind ions such as $\mathrm{Fe}^{+6}$ are required. These corrections have not yet been applied to the distribution function of heavy pickup ions shown here. Note the similarity in the shapes of the inner source spectrum of $\mathrm{O}^{+}$shown here and that of $\mathrm{H}^{+}$given by the dashed curve of Figure 2.

terstellar pickup $\mathrm{O}^{+}$(the dotted model curve) has been subtracted. The two spectra $\left(\mathrm{O}^{+}\right.$and $\left.\mathrm{C}^{+}\right)$are nearly identical within experimental uncertainties, and both reach maximum phase-space density of a few s $\mathrm{sm}^{-6}$ at $W$ of about 0.85 . This suggests a common source for these two heavy pickup ions, a source that supplies almost as much oxygen as carbon. Because the phase-space densities increase with decreasing $W$ to peak just below $W=1$, the source for these pickup $\mathrm{C}^{+}$and $\mathrm{O}^{+}$must be located much closer to the Sun than the 5 AU distance of Ulysses during these observations. Peaked distributions would be expected to be the result of adiabatic cooling of ions picked up far away from where they are detected (Vasyliunas and Siscoe, 1976; Gloeckler et al., 1993; Gloeckler, 1996; Fisk et al., 1997).

We have begun to investigate the characteristics of this inner source (or inner sources), located between Ulysses and the Sun, by measuring the velocity distributions of heavy pickup ions at different positions of the spacecraft. The mass/charge histogram of heavy, singly charged ions with $\mathrm{W}$ between 0.8 and 1.0 observed 


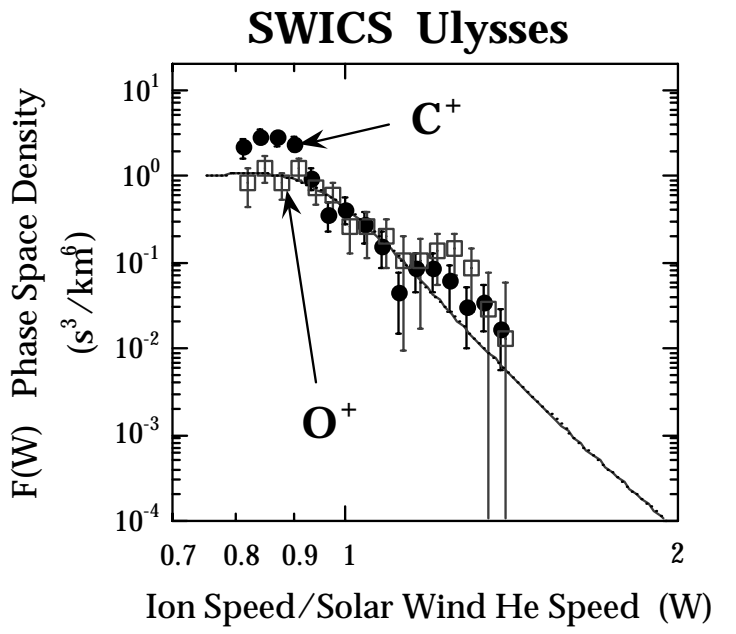

Figure 11. Same as Figure 10 except for $\mathrm{O}^{+}$, from which the interstellar contribution represented by the dotted curve of Figure 10 has been subtracted, and $\mathrm{C}^{+}$. Within the statistical errors the two spectra are about the same. The somewhat higher density of $\mathrm{C}^{+}$at $W \sim 0.85$ may indicate a higher production of pickup carbon compared to oxygen (Rucinski et al., 1996) from a source that produces comparable densities of atomic $\mathrm{C}$ and $\mathrm{O}$. The smooth curve is the inner source spectrum represented by the dashed curve of Figure 10. Integrating the inner source spectrum of $\mathrm{C}^{+}$(assumed to be the same as for $\mathrm{O}^{+}$) and combining this with the $\mathrm{O}^{+}$spectrum of Figure 10 gives a total $\mathrm{C}^{+} / \mathrm{O}^{+}$ratio of 0.003 above $W \approx 1.4$.

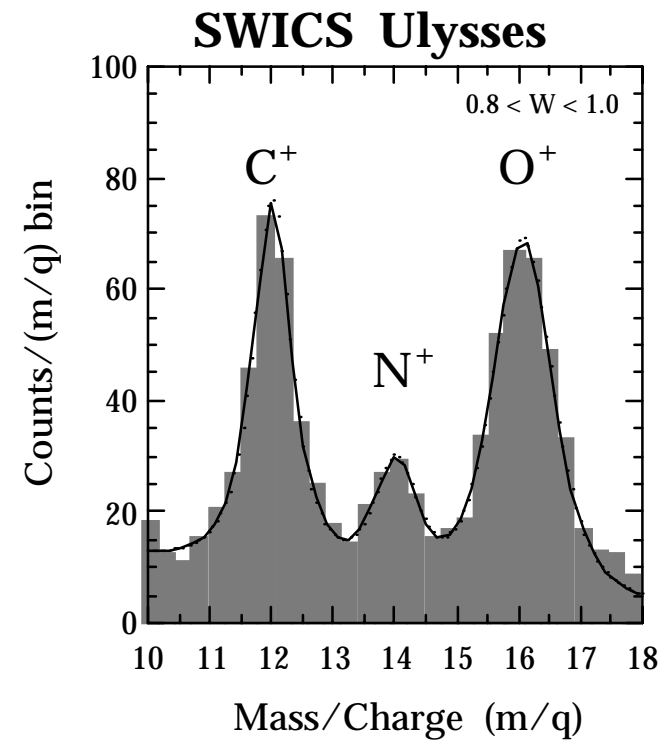

Figure 12. Plot of the double coincidence counts per mass/charge $(m / q)$ interval of ions with W between 0.8 and 1.0 versus the mean $m / q$ of each interval. These SWICS data were accumulated during all of 1994 at an average distance of $2.8 \mathrm{AU}$ from the Sun and average helio-latitude of $-65^{\circ}$. The average solar wind helium speed was $784 \mathrm{~km} \mathrm{~s}^{-1}$. In addition to the ${ }^{16} \mathrm{O}^{+}$and ${ }^{14} \mathrm{~N}^{+},{ }^{12} \mathrm{C}^{+}$is now observed at a level comparable to ${ }^{16} \mathrm{O}^{+}$. 


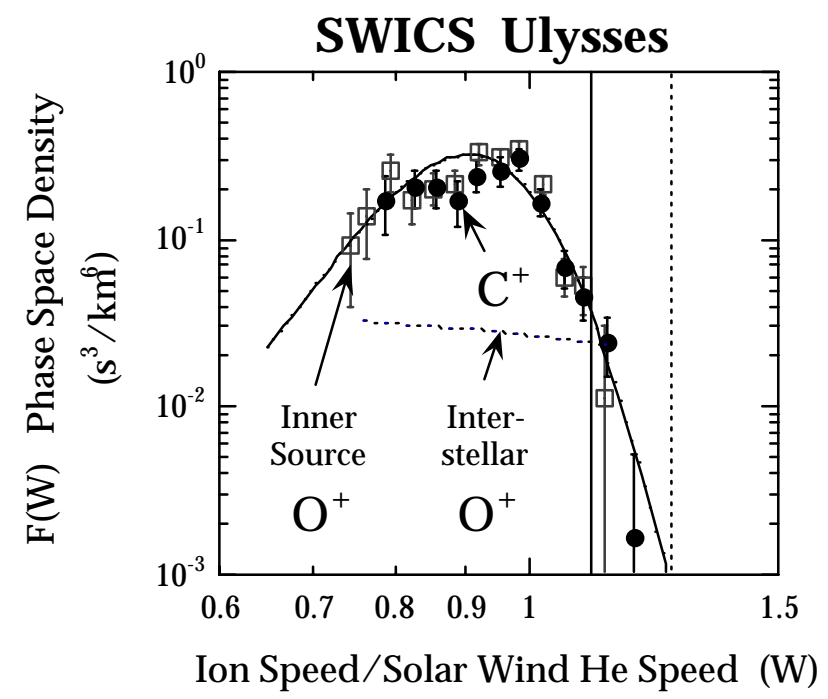

Figure 13. Phase-space density of $\mathrm{C}^{+}$and $\mathrm{O}^{+}$versus $\mathrm{W}$ during all of 1994 when Ulysses was at high latitudes at an average distance of $2.8 \mathrm{AU}$. The vertical solid and dashed lines indicate the average upper $\mathrm{W}$ cutoff for $\mathrm{O}^{+}$and $\mathrm{C}^{+}$, respectively, above which the SWICS instrument upper energy per charge limit of $60 \mathrm{keV} \mathrm{e}^{-1}$ prevents detection of these singly charged heavy pickup ions in the high speed solar wind. The dashed curve is the expected contribution of interstellar $\mathrm{O}^{+}$. It is evident that in the high speed stream interstellar $\mathrm{O}^{+}$is entirely obscured by the far more abundant $\mathrm{O}^{+}$from the inner source. The solid curve represents a hypothetical inner source spectrum. The shape of the inner source distribution viewed here from high latitudes is somewhat different from that observed in the ecliptic plane (Figure 11). This difference is most likely due to different spatial profiles of this distributed inner source in and out of the ecliptic. See text for further discussion.

during 1994, when Ulysses was in the high-speed solar wind and at high latitudes, is shown in Figure 12. In contrast to what was seen at low latitude and high $W$ (cf., Figure 9), $\mathrm{C}^{+}$is now present at a level comparable to $\mathrm{O}^{+}$. A peak at $m / q=14$ is also clearly visibly. It is unlikely that this peak is due predominantly to solar wind $\mathrm{Fe}^{+4}$ because no significant amount of the more abundant $\mathrm{Fe}^{+5}$ is observed at $m / q=11.2$.

The distribution functions of $\mathrm{C}^{+}$and $\mathrm{O}^{+}$for this one-year period, when Ulysses was at high latitudes, are shown in Figure 13. Because of the high solar wind speed $\left(\left\langle V_{\mathrm{sw}}\right\rangle=780 \mathrm{~km} \mathrm{~s}^{-1}\right)$ the SWICS energy per charge upper limit of $60 \mathrm{keV} \mathrm{e}^{-1}$ prevented detection of $\mathrm{C}^{+}$and $\mathrm{O}^{+}$beyond $W$ of 1.26 and 1.1, respectively (dotted and solid vertical lines). This instrumental limit excludes interstellar $\mathrm{O}^{+}$which otherwise would be visible beyond $W \sim 1.1$ as is indicated by the model curve (labeled Interstellar $\mathrm{O}^{+}$). The velocity distributions of $\mathrm{C}^{+}$and $\mathrm{O}^{+}$in the observable $W$ range are identical within experimental errors but have rather complicated shapes. The observed phase-space densities decrease modestly with decreasing $W$ below $\sim 0.95$ but fall rapidly above $W \sim 0.95$. Concentration of density around $W$ close to 1 again suggests that the source of these heavy pickup ions again lies well 


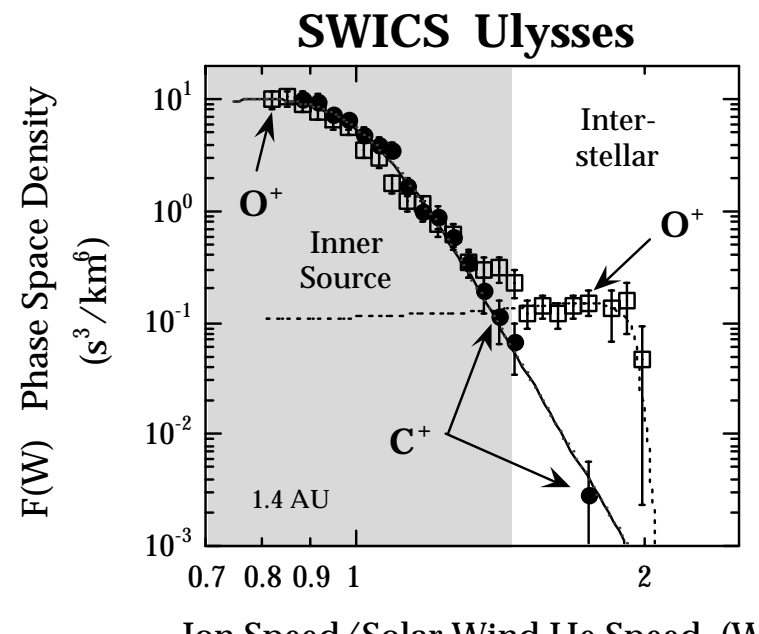

Ion Speed/Solar Wind He Speed (W)

Figure 14. Average phase-space density of $\mathrm{C}^{+}$and $\mathrm{O}^{+}$versus $\mathrm{W}$ during the fast pole-to-pole pass of Ulysses through the ecliptic plane (5 February to 29 March 1995). Only data for slow solar wind speed $\left(<550 \mathrm{~km} \mathrm{~s}^{-1}\right)$ were included. The average speed for the selected data was $444 \mathrm{~km} \mathrm{~s}^{-1}$ and the average distance of Ulysses was 1.4 AU from the Sun. The dashed curve represents the expected contribution of interstellar $\mathrm{O}^{+}$. Despite the dominance of the inner source at $W$ less than about 1.5 interstellar pickup oxygen is still seen above $W=1.5$. The solid curve represents a hypothetical inner source phase-space density distribution. Above $W \approx 1.5$ little if any $\mathrm{C}^{+}$appears to be of interstellar origin. Notice the similarity in the shape of the inner source spectrum shown here to that given in Figure 11.

within the average radial distance of Ulysses of 2.8 AU during 1994 when these data were taken.

The rapid pole-to-pole passage of Ulysses in 1995 provided the opportunity to observe pickup ion distributions when Ulysses was again in the ecliptic plane but now only 1.4 AU away from the Sun. Because much of this time the solar wind speed was well below $\sim 600 \mathrm{~km} \mathrm{~s}^{-1}$, the distribution functions of $\mathrm{C}^{+}$and $\mathrm{O}^{+}$over the entire speed range up to $W=2$ could be measured. The $\mathrm{C}^{+}$and $\mathrm{O}^{+}$spectra we observed are shown in Figure 14. The mass/charge histogram for heavy ions with $W$ between 0.9 and 1.5 for this same time period is given in Figure 15. The distribution functions of $\mathrm{C}^{+}$and $\mathrm{O}^{+}$are again identical (within errors) and the phase-space density is decreasing with increasing $\mathrm{W}$ from a maximum of $\sim 10 \mathrm{~s}^{3} \mathrm{~km}^{-6}$ at $W \sim 0.85$. The decrease in the density is not as rapid as for the spectra of Figure 13, when Ulysses was at high latitude and about twice as far away from the Sun, indicating an inner source close to the Sun extending not much beyond $1 \mathrm{AU}$. For $\mathrm{O}^{+}$the spectrum becomes flat for $W>\sim 1.5$ with a cutoff at $W=2$. The phase-space density in this high $W$ interval is entirely consistent with oxygen of interstellar origin and an atomic oxygen density of $7.8 \times 10^{-5} \mathrm{~cm}^{-3}$ at the termination shock. Thus, as close as $1.5 \mathrm{AU}$ and perhaps even at $1 \mathrm{AU}$ where the ACE spacecraft is positioned, observations of interstellar pickup oxygen should 


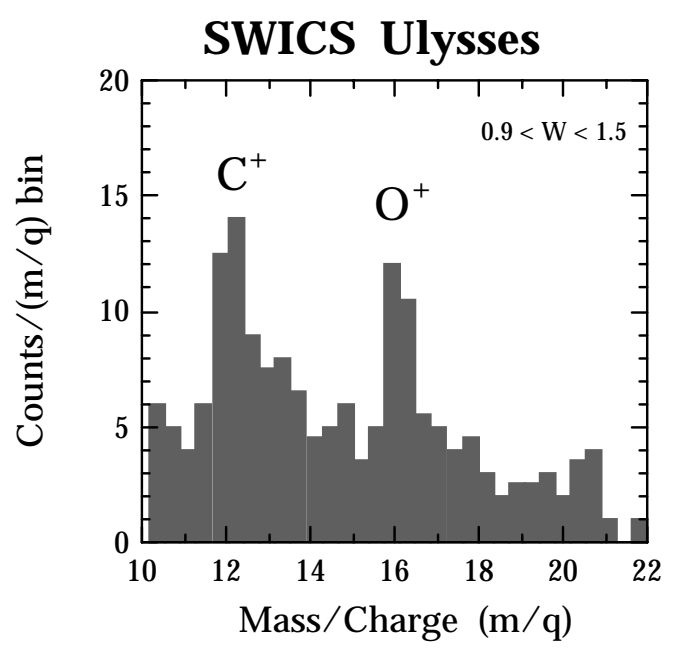

Figure 15. Plot of the double coincidence counts per mass/charge $(m / q)$ interval of ions with W between 0.9 and 1.5 versus the mean $m / q$ of each interval. These SWICS data were accumulated for the same period and selection criteria as given in the caption to Figure $14 .{ }^{16} \mathrm{O}^{+}$and ${ }^{12} \mathrm{C}^{+}$are observed at comparable levels. Other elements or molecules may be present but cannot be resolved in this data set.

be possible since it appears to be not entirely obscured by the $\mathrm{O}^{+}$from the inner source.

The distribution functions of the inner source pickup oxygen and carbon are complicated and not well understood. If one accepts adiabatic energy losses then the peaked distributions indicate a source located predominately upstream of Ulysses, very likely within a few AU from the Sun. The phase-space densities of both $\mathrm{O}^{+}$and $\mathrm{C}^{+}$peak consistently somewhat below $W=1$. We cannot find any instrumental or analysis effects that would cause the peaks of these distributions to shift to slightly below $W=1$. One way to interpret the inner source spectra is to postulate weak scattering at all pitchangles in regions close to the Sun (i.e., $<1 \mathrm{AU}$ ) where, in the primarily radial magnetic field, most of these pickup ions are created with average speeds of $W$ less than 1 . Adiabatic cooling would still take place due to conservation of the first adiabatic invariant in the diverging magnetic field. The cooling, however, would be primarily perpendicular to the magnetic field. While this could account in a qualitative manner for the peaked spectra of the inner source ions, much work still remains to be done to fully understand these observations and to make firm inferences concerning the spatial distribution of the inner source material. 


\section{Discussion and Conclusions}

The capability to measure mass/charge, mass, and speed of an ion, and the low background of the SWICS instrument combined with the trajectory of Ulysses make it possible to distinguish ions originating from the Sun, the interstellar gas, and the newly discovered inner source (cf., Geiss et al., 1995, 1996). The singly charged suprathermal interstellar pickup ions can be easily identified and separated from the far more abundant solar wind ions which are multiply charged and have relatively narrow velocity distributions peaking at the solar wind bulk speed. This has enabled us to study pickup ions to an extent never possible before, and to draw numerous conclusions regarding their origin and behavior, and the characteristics of the regions of their origin.

\subsection{INTERACTION OF PICKUP IONS WITH THE SOLAR WIND}

The velocity distributions of interstellar pickup hydrogen and helium observed in the steady and relatively unperturbed solar wind of the polar coronal hole provide clear evidence for lack of significant thermalization and energy diffusion. However, while adiabatic cooling is unmistakable, the strongly anisotropic distributions in the predominantly radial field at high latitudes indicate weak pitch-angle scattering. To reconcile these two apparently conflicting results one can imagine two extreme cases: (a) pitch-angle scattering is suppressed only at $90^{\circ}$ pitch-angles (Fisk et al., 1997) or (b) pitch-angle scattering is weak at all pitch-angles and cooling results only perpendicular to the magnetic field (Möbius, 1996). In either case modeling of the expected evolution of the pickup ion distribution function is complicated and has as yet not been accomplished. In a magnetic field predominately perpendicular to the solar wind flow direction, nearly isotropic distributions (i.e., equal density below and above $W=1$ ) are observed (Gloeckler et al., 1997) and are expected in either of the two cases. Fisk et al. (1997) find that weak pitch-angle scattering at $90^{\circ}$ is most consistent with SWICS observations of interstellar pickup hydrogen.

A consequence of weak pitch-angle scattering is the observed correlation between fluctuations of the phase-space densities of pickup hydrogen and helium, both integrated between $W=1.6$ and 2.0, shown in Figure 16 (Fisk et al., 1997). This initially surprising correlation is quite likely due to the variation of the mean free path for scattering through $90^{\circ}$ which controls and modulates the densities of both pickup $\mathrm{H}^{+}$and $\mathrm{He}^{+}$in the $W>\sim 1.6$ range. Calculations of the dependence of the fractional density fluctuations, $\Delta n / n$, on scattering mean free path $(\lambda)$ variations, $\Delta \lambda / \lambda$, show that, in order to be consistent with observations, a large mean free path $(\lambda \sim 1 \mathrm{AU})$ is required (Fisk et al., 1997) for reasonable $\Delta \lambda / \lambda(<\sim 1)$.

We have interpreted interstellar pickup ion spectra assuming weak pitch-angle scattering only at $90^{\circ}$ pitch-angle, and strong scattering on either side of $90^{\circ}$. It is then possible to model $\mathrm{H}^{+}$and $\mathrm{He}^{+}$and $\mathrm{He}^{++}$distribution functions and obtain in a self-consistent fashion the respective ionization loss rates for atomic $\mathrm{H}$ and $\mathrm{He}$ in 


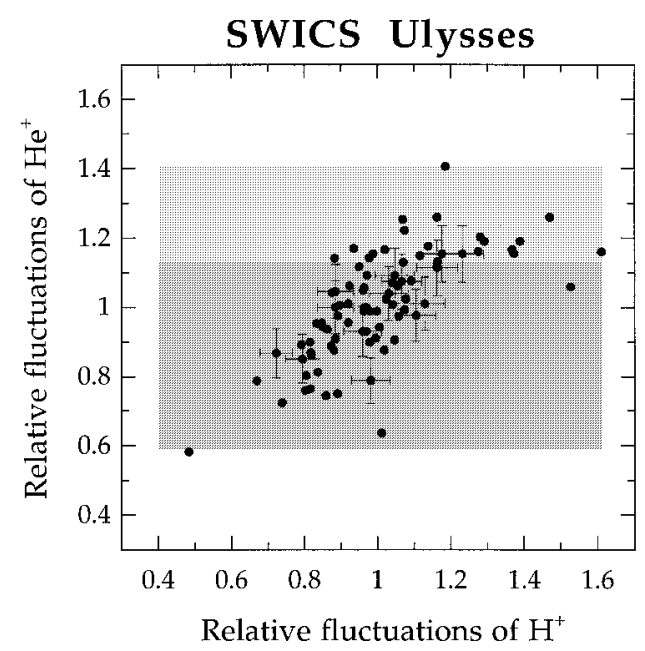

Figure 16. The fractional density fluctuations of interstellar pickup $\mathrm{H}^{+}$versus those of interstellar pickup $\mathrm{He}^{+}$. Each data point represents a 2-day average of the respective integrated phase-space density from $W=1.6$ to 2.0 divided by the corresponding 30 -day smoothed density. These observations were made between January 1 and June 30, 1994 when Ulysses was in the high speed stream (770 to $790 \mathrm{~km} \mathrm{~s}^{-1}$ ) of the southern polar coronal hole. The helio-centric distance of Ulysses ranged from 2.88 to $3.8 \mathrm{AU}$ during this period.

the heliosphere as well as atomic abundances at the heliospheric termination shock. The ionization rates we determined from the shapes of the pickup ion spectra were found to be consistent with independently derived rates (Rucinski et al., 1996), and our values for the atomic $\mathrm{H}$ and $\mathrm{He}$ densities agreed well with those derived from UV backscatter observations (Quemerais et al., 1996) for $\mathrm{H}$ and from direct detection of neutral He on Ulysses (Witte et al., 1993, 1996).

In the disturbed solar wind, as is the case in co-rotating interaction regions between the forward and reverse shocks, the distribution functions of pickup as well as solar wind ions become broader with pronounced tails extending to high energies (cf., Figures 7 and 8). Clearly, pickup $\mathrm{H}^{+}$and $\mathrm{He}^{+}$are easily accelerated in these regions. Less certain is the proportion of pickup as opposed to solar wind ions that is accelerated. Just how much of each of these two populations is accelerated in CIRs will very likely depend on the relative fraction of these populations in the suprathermal range, perhaps around $W \approx 2$. Since the solar wind generally is heated more behind the reverse shock than it is behind the forward shock (WimmerSchweingruber et al., 1997) one would expect that ions accelerated at the reverse shock are predominantly solar wind ions (see Figure 8). On the other hand, when the solar wind speed is high, pickup ion distributions extend to higher speeds, and at twice the solar wind speed they may again have densities comparable to or higher than those of solar wind ions heated to these higher speeds. It seems to be the case that in CIRs at $\sim 3-5 \mathrm{AU}$, the heated solar wind distributions for $\mathrm{H}, \mathrm{He}, \mathrm{C}$, and $\mathrm{O}$ and the corresponding pickup ion distributions have roughly comparable 
densities near $W \sim 2$ and therefore relatively small changes in either the solar wind thermal speed or its bulk speed will affect the proportion of solar wind versus pickup ions accelerated in CIRs. Observations of the larger relative abundance of $\mathrm{H}$ and He of energetic particles in CIRs compared to that in the solar wind (Gloeckler et al., 1979; Reames et al., 1991) support our conclusion that pickup ions form a significant part of the seed population of energetic particles accelerated in these turbulent regions.

The inner source could also contribute substantially to the energetic particles accelerated in co-rotating interaction regions (cf., Geiss et al., 1995, 1996). The average $\mathrm{C} / \mathrm{O}$ ratio of CIR accelerated particles is higher than the solar wind ratio (Gloeckler et al., 1979; Reames et al., 1991; Fränz et al., 1995) but close to the $\mathrm{C} / \mathrm{O}$ ratio of about 1 observed in the inner source. However, we would again expect that the proportion of inner source pickup ions versus solar wind $\mathrm{C}$ and $\mathrm{O}$ that is accelerated will be very sensitive to the thermal and bulk speed of the ambient solar wind.

To what extent heliospheric acceleration of pickup ions, as shown here, contributes to the production of anomalous cosmic rays (ACR) remains an open question. At the very least some pre-acceleration of pickup ions inside the heliosphere is required in order to compensate for adiabatic cooling. In the absence of this acceleration, pickup ions would be adiabatically cooled, as is observed, and at the termination shock their phase-space density would be more concentrated around $W=1$, with smaller density near the cutoff at $W=2$ and very few pickup ions beyond the $W=2$ cutoff. At the other extreme, heliospheric acceleration may occur not only in the inner ( $\sim 3$ to $\sim 10$ AU) heliosphere as is observed, but also at large distances from the Sun at latitudes not yet explored. One possibility, suggested by Fisk (1996) is acceleration even at distances far beyond $\sim 10 \mathrm{AU}$ at the interface between the low speed flow in the ecliptic and the high speed flow from the polar coronal holes at higher latitudes. Presumably, pickup ions in the turbulence associated with this interface would be accelerated as efficiently as in CIRs. Finally, we note that because little atomic $\mathrm{C}$ exists in the local interstellar cloud (Frisch, this issue) only a small amount of interstellar carbon is available for acceleration inside the termination shock. Thus, much of the ACR carbon may come from the inner source (Geiss et al., 1996), requiring then substantial heliospheric acceleration to overcome the drastic adiabatic cooling of inner source pickup carbon.

\subsection{COMPOSITION OF THE LOCAL INTERSTELLAR CLOUd (LIC)}

The absolute number densities of atomic $\mathrm{H}, \mathrm{He}, \mathrm{N}, \mathrm{O}$, and $\mathrm{Ne}$ in the interstellar gas flowing through the heliosphere can be obtained without much ambiguity from measurements of the phase-space densities of the corresponding pickup ions. For $\mathrm{H}$ and $\mathrm{He}$ there are no significant contributions from possible inner sources of these atoms, and even for $\mathrm{N}$ and $\mathrm{O}$ the interstellar gas ions can be well separated from 
inner source ions (cf., Geiss et al., 1995, 1996) as is best demonstrated by the phasespace density spectra (cf., Figures 10 and 14). Thus, at distances greater than $5 \mathrm{AU}$, less than a few percent of the $\mathrm{O}^{+}$ions in the velocity range $W>1.4$ come from the inner source (see Figures 9 and 11). Since the interstellar ion abundances of $\mathrm{N}^{+}, \mathrm{O}^{+}$ and $\mathrm{Ne}^{+}$given by Geiss et al. (1994a) were essentially based on ions with $W>1.3$ near Ulysses' aphelion, no significant corrections for an inner source contribution are needed for the interstellar gas abundances reported there, even though these abundances were obtained before the inner source was discovered.

We have used our measurements of the velocity distributions of pickup ions (such as shown in Figures 2, 3, 5, and 10) to derive the atomic number densities of ${ }^{1} \mathrm{H},{ }^{4} \mathrm{He},{ }^{3} \mathrm{He},{ }^{14} \mathrm{~N},{ }^{16} \mathrm{O}$, and ${ }^{20} \mathrm{Ne}$ at the termination shock $(\sim 100 \mathrm{AU})$. The ratios (relative to ${ }^{4} \mathrm{He}$ ) and the number densities are given in columns 2 and 3 , respectively, of Table I. The ${ }^{1} \mathrm{H}$ and ${ }^{4} \mathrm{He}$ values are from Gloeckler et al. (1997), the ${ }^{3} \mathrm{He}$ abundance is taken from Gloeckler and Geiss (1998). The interstellar heavy element abundances are derived from SWICS measurements of $\mathrm{O}^{+}$reported here (cf., Figures 9 and 10) and from similar distribution functions of $\mathrm{N}^{+}$and $\mathrm{Ne}^{+}$(not shown). The errors given next to the values include estimates of systematic errors in the ionization rates of the respective species. These systematic errors are in general the largest source of uncertainty.

In the local interstellar cloud (LIC) matter exists in the form of atoms, ions and grains (or dust). Only atoms and very low-charged grains can enter the heliosphere as indicated in Figure 1. For $\mathrm{H}, \mathrm{O}$, and $\mathrm{N}$ there is a reduction in the density of atoms reaching the termination shock compared to their abundance in the LIC due to charge-exchange ionization with the slow moving (compared to neutrals) interstellar protons in the filtration region (roughly 100-200 AU wide) just beyond the heliopause (Holzer, 1989; Baranov and Malama, 1995; Gloeckler et al., 1997). Because of the small charge-exchange cross section for noble gases, $\mathrm{He}$ and $\mathrm{Ne}$ are not affected by this process. Using SWICS measurements of pickup ions $\left(\mathrm{H}^{+}\right.$ and $\mathrm{He}^{++}$) Gloeckler et al., (1997) have found the amount of reduction (filtration) for hydrogen and from this computed the fractional ionization for $\mathrm{H}$ and $\mathrm{He}$ in the LIC. The derived densities in the LIC of atoms and ions are given in columns 4 and 5 of Table I.

The $\mathrm{O} / \mathrm{He}$ ratio at the termination shock derived from the phase-space density data shown in Figures 10 and 2 falls well within the error limits of our earlier value. The uncertainties of the present ratio are smaller, because of the availability of a larger data base of slow solar wind measurements at large solar distance, and a better knowledge of the ionization rates. Geiss et al. (1994a) included in their error limits large uncertainties in the production and loss rates for oxygen.

The depletion of atomic oxygen in the heliosphere caused by filtration in the interaction region beyond the heliopause can be estimated by comparing our oxygen density at the termination shock $\left(7.8 \times 10^{-5} \mathrm{~cm}^{-3}\right)$ with the atomic oxygen density in the LIC. The LIC density of $\mathrm{O}$ is derived by combining the LIC HI/OI ratio, determined to be 2090 by Linsky et al. (1995), with our estimate of the atomic hy- 
TABLE I

Densities of atoms at the heliospheric termination shock ( $~ 85-110 \mathrm{AU})$ and densities of atoms and ions in the local interstellar cloud (LIC) of elements and isotopes measured as pickup ions with SWICS on Ulysses

\begin{tabular}{|c|c|c|c|c|c|c|c|}
\hline \multirow[t]{3}{*}{ Isotope } & \multicolumn{2}{|c|}{ Termination shock } & \multicolumn{4}{|c|}{ Local Interstellar Cloud (LIC) } & \multirow{3}{*}{$\begin{array}{l}\text { Solar system } \\
\text { abundance } \\
\text { ratio }\end{array}$} \\
\hline & \multirow[t]{2}{*}{ Ratio } & \multirow[t]{2}{*}{ Density $\left(\mathrm{cm}^{-3}\right)$} & \multicolumn{3}{|l|}{ Density* } & \multirow[t]{2}{*}{ Ratio } & \\
\hline & & & Atoms & Ions & Total & & \\
\hline${ }^{1} \mathrm{H}$ & 7.5 & $0.115 \pm 0.025$ & 0.20 & 0.043 & 0.243 & 10 & 10 \\
\hline${ }^{4} \mathrm{He}$ & 1.000 & $0.0153 \pm 0.0018$ & 0.0153 & 0.0090 & 0.0243 & 1 & 1 \\
\hline${ }^{3} \mathrm{He}$ & $2.48 \times 10^{-4}$ & $(3.8 \pm 1.0) \times 10^{-6}$ & $3.8 \times 10^{-6}$ & $2.2 \times 10^{-6}$ & $6.0 \times 10^{-6}$ & $2.5 \times 10^{-4}$ & - \\
\hline${ }^{14} \mathrm{~N}$ & $0.6 \times 10^{-3}$ & $(0.92 \pm 0.28) \times 10^{-5}$ & $1.0 \times 10^{-5}$ & $2.2 \times 10^{-6}$ & $1.2 \times 10^{-5}$ & $5.1 \times 10^{-4}$ & $1.12 \times 10^{-3}$ \\
\hline${ }^{16} \mathrm{O}$ & $5.1 \times 10^{-3}$ & $(7.8 \pm 1.4) \times 10^{-5}$ & $9.5 \times 10^{-5}$ & $2.1 \times 10^{-5}$ & $1.2 \times 10^{-4}$ & $4.8 \times 10^{-3}$ & $8.51 \times 10^{-3}$ \\
\hline${ }^{20} \mathrm{Ne}$ & $0.75 \times 10^{-3}$ & $(1.15 \pm 0.25) \times 10^{-5}$ & $1.15 \times 10^{-5}$ & $6.7 \times 10^{-6}$ & $1.8 \times 10^{-5}$ & $7.5 \times 10^{-4}$ & $1.23 \times 10^{-3}$ \\
\hline
\end{tabular}

${ }^{*}$ For nitrogen and oxygen we asumed the same ionization fraction was measured for hydrogen $(0.18)$. For Ne a the same ionization fraction as determined for $\mathrm{He}(0.37)$ was assumed. 
drogen density of $0.20 \mathrm{~cm}^{-3}$ in the local cloud (column 4 of Table I). We arrive at an $\sim 18 \%$ loss of atomic oxygen by filtration using the estimated LIC atomic oxygen density of $9.5 \times 10^{-5} \mathrm{~cm}^{-3}$ and our measured oxygen density at the termination shock. The loss of $\mathrm{H}$ by filtration is about $42 \%$ (Gloeckler et al., 1997). Thus, the observed filtration losses of $\mathrm{H}$ and $\mathrm{O}$ are consistent with the theoretical predictions of Geiss et al. (1994b) who showed that under all conditions the filtration loss caused by charge exchange is smaller for $\mathrm{O}$ than for $\mathrm{H}$. Since the charge-exchange cross section between nitrogen and hydrogen is smaller than between oxygen and hydrogen, and since the ionization energy of $\mathrm{N}$ is larger than that of $\mathrm{O}$, the loss by filtration of $\mathrm{N}$ should be even smaller than that of $\mathrm{O}$. For the LIC abundance given in Table I we therefore assume a small N loss (10\%) due to filtration.

The ionization fractions of the high FIP (first ionization potential) $\mathrm{N}$, O, and $\mathrm{Ne}$ are not well known, but believed to be far less than for low FIP elements (e.g., $\mathrm{C}, \mathrm{Mg}, \mathrm{Fe}$ ) which are highly ionized (e.g., Frisch, this issue). We have assumed the same ionization fraction for $\mathrm{N}$ and $\mathrm{O}$ as for hydrogen $(0.18)$ and the same ion fraction of $\mathrm{Ne}$ as for $\mathrm{He}(0.37)$ to compute the ion densities and total densities of $\mathrm{N}, \mathrm{O}$, and $\mathrm{Ne}$ in the Local Interstellar Cloud. In comparing the abundance ratios (relative to ${ }^{4} \mathrm{He}$ ) of elements measured in the LIC (column 7 of Table I) to those in the protosolar nebula given in column 8 (Anders and Grevesse, 1988), we find that nitrogen and oxygen are underabundant in the LIC by about a factor of 2 and $\mathrm{Ne}$ by about 1.6. The abundance of $\mathrm{N}, \mathrm{O}$ and other elements in B-stars is about $50 \%$ lower than in the Sun (e.g., Frisch, this issue). Our results suggest that the composition in the LIC may be better represented by B-star rather than solar abundances. Using B-star rather than solar abundances our results further suggest that about $15 \%$ of the total oxygen abundance in the LIC may be in the form of grains.

\subsection{LOCATION OF THE HELIOSPHERIC TERMINATION SHOCK}

Observations of pickup ions were used by Gloeckler et al. (1997) to estimate the minimum distance to the termination shock. Assuming pressure balance at the heliopause, measurements of atomic densities and ion fractions in the LIC were used to calculate the position of the termination shock as a function of the magnetic field strength in the LIC. A value of $1.6 \mu \mathrm{G}$ for the magnetic field (Frisch, 1996) places the shock between 75 and 95 AU. Lack of detection of the shock by Voyager 1 at its present location close to $70 \mathrm{AU}$ restricts the field to an upper limit of $\sim 4 \mu \mathrm{G}$, a value insufficient to pressure balance the hot large cloud in which the LIC is embedded. To provide the required balance Gloeckler et al.,(1997) suggested that either the magnetic field in the LIC is inhomogeneous or that there is a significant and, as yet undetected, non-thermal component to the pressure in the local cloud. 


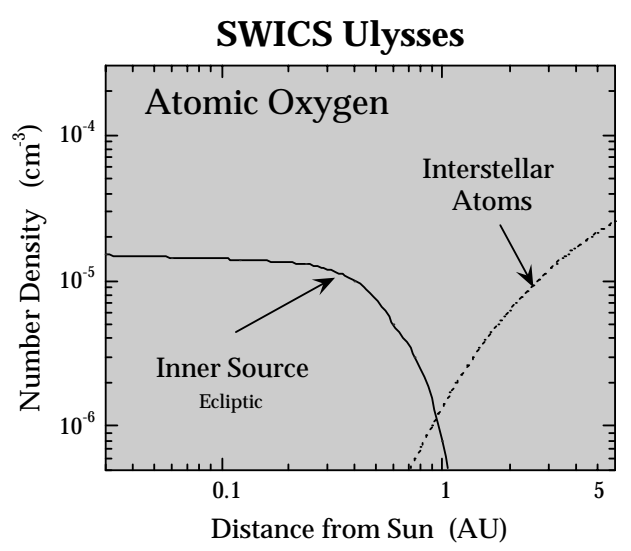

Figure 17. Dependence of the density of atomic oxygen from the inner and interstellar sources on radial distance. The dotted curve, representing the interstellar atomic oxygen, has been computed with the same ionization rates used for the model fit of $\mathrm{O}^{+}$shown in Figure 10. The solid curve represents a sketch of the radial density profile of the inner source in the ecliptic plane. This spatial distribution is roughly consistent with the spectral shape of the inner source oxygen shown in Figure 14. Statistical uncertainties of the data used and poor knowledge of the evolution of pickup ion distributions do not allow us to exclude more complex radial profiles for the inner source.

\subsection{THE EXTENDED INNER SOURCE OF PICKUP IONS}

Densities of pickup ions produced by the inner source are expected to decrease approximately with $r^{2}$ at heliocentric distances sufficiently beyond the source region. The distance of maximum density of interstellar gas pickup ions, on the other hand, scales roughly with their ionization rate. For $\mathrm{H}^{+}, \mathrm{N}^{+}$, and $\mathrm{O}^{+}$this maximum is at or beyond the aphelion distance of Ulysses (see Figure 6). There is also a striking difference in the velocity distributions of the two pickup ion populations, as is demonstrated in Figures 10, 11, and 14. Once the solar wind has left the region of the inner source, no new pickup ions from this source are created and the velocity distributions of these ions become progressively narrower with increasing distance due to adiabatic cooling, and therefore, the cutoff speed moves away from $W=2$ toward $W=1$. Closer to the Sun and for $W<\sim 1.5$, the contribution by the inner source to the total pickup ion population is substantial, as the relatively high abundance of $\mathrm{C}^{+}$in Figure 14 indicates. Nevertheless, the total production in the heliosphere of inner source ions is much smaller than the production of ions from the interstellar gas (cf., Table 1 in Geiss et al., 1996).

The nature of the inner source of pickup ions is at present not so readily specified. This source seems to produce ions over a range of solar distances and over virtually all solar latitudes. In Figure 17 we show a sketch of the approximate density profile for atomic oxygen from the inner source viewed from the ecliptic at 1.4 AU (solid curve) as well as the spatial density profile of the interstellar source (dotted curve). Our determination of the inner source radial profile (from the distribution function shown in Figure 14) is subject to uncertainties due to our poor 
knowledge of evolution of the pickup ion distributions. Nevertheless, this density profile can be used to estimate the total production rate of oxygen from the inner source to be $\sim 7 \times 10^{6} \mathrm{~g} \mathrm{~s}^{-1}$. The production rate for carbon is comparable since roughly equal amounts of $\mathrm{C}^{+}$and $\mathrm{O}^{+}$are found in the inner source (cf., Figures 13 and 14). This suggests that the contribution of the inner source to the production of anomalous cosmic-ray carbon (at a rate of $\sim 10^{7} \mathrm{~g} \mathrm{~s}^{-1}$ estimated by Geiss et al., 1996) may be important.

Present observations of the phase-space densities of carbon and oxygen (cf., Figures 11, 13, and 14) cannot be used to rule out the existence of more than one inner source of pickup ions. Our observations suggest that the extended inner source is likely to be complex, with different source materials and different ion production mechanisms contributing. In our judgment, the most important of these are:

(1) Thermal release of molecules from interstellar grains passing through the heliosphere. The source strength is not expected to depend strongly on latitude. The radial dependence of densities shows that for $\mathrm{C}^{+}$and $\mathrm{O}^{+}$, the mechanism of release cannot be temperature-independent sputtering.

(2) Thermal release of molecules from interplanetary grains. This source is expected to be concentrated near the ecliptic plane.

(3) Small comets. Whereas medium and large comets would appear to SWICS/ Ulysses as point sources (cf., Geiss et al., 1996), a large number of small comets ('mini-comets') could not be distinguished from a truly distributed source.

(4) Collisions of grains. Grün et al. (1985) have shown that, inside the orbit of earth, collisions become the main loss mechanism for grains on PoyntingRobertson trajectories. Due to solar radiation pressure, small fragments created by these collisions will assume hyperbolic orbits (beta meteoroids) and escape rapidly, but some of the material will evaporate locally and subsequently ionize, forming pickup ions.

(5) Solar wind ions absorbed by grains and desorbed as atoms and molecules. Pickup ions are then created from this solar material through re-ionization by solar EUV and solar wind charge exchange.

All these sources depend on the existence of small solid objects in the solar wind, upstream of Ulysses. The initial velocity distributions of pickup ions created from these sources are virtually the same, but they evolve with distance from the point of origin by pitch-angle scattering, adiabatic cooling and perhaps other processes. The composition of sources 1 to 4 reflects the composition of components in the solids. However, interstellar and interplanetary grains are likely to have very inhomogenous compositions, so that even a single grain population could produce a complex source pattern.

Source 5 creates pickup ions of solar origin. The underlying mechanism has been discussed by various authors (e.g., Banks, 1971; Fahr et al., 1981; Gruntman, 1996) in the context of producing atomic and molecular hydrogen, as well as $\mathrm{H}^{+}$ and $\mathrm{H}_{2}{ }^{+}$pickup ions. This mechanism will of course also produce heavier pickup 
ions, such as $\mathrm{C}^{+}$and $\mathrm{O}^{+}$and $\mathrm{Ne}^{+}$. The source strength will depend on the grain density in the upstream region. Grains on Poynting-Robertson trajectories will be more effective than grains on transient orbits (interstellar grains, beta-meteoroids), because the latter spend only a relatively short time near perihelion where solar wind fluxes and temperatures are high.

In order to quantify the relative contributions of these five sources, a comprehensive investigation of the phase-space densities of $\mathrm{H}^{+}, \mathrm{He}^{+}, \mathrm{C}^{+}, \mathrm{N}^{+}, \mathrm{O}^{+}, \mathrm{Ne}^{+}$, and other species as a function of heliospheric latitude, longitude and distance from the Sun is needed. This can be achieved by using the data of SWICS/Ulysses, supplemented by data from STICS/WIND, CELIAS/SOHO and especially SWICS/ACE. It is also essential to understand far better than we do now the evolution of the pickup ion distribution functions and the scattering properties of these ions. Such work is now in progress. Comparing the results with measured and inferred densities of interstellar and interplanetary grains, and with observations relevant to the possible existence of mini-comets (cf., Brandt et al., 1996) should enable us to better specify the relative contributions to the inner source.

\section{Acknowledgments}

We are very grateful to the many individuals (see Gloeckler et al., 1992) at the University of Maryland, the University of Bern, the Max-Planck-Institute für Aeronomie and the Technical University of Braunschweig for their many outstanding contributions to the success of the SWICS experiment on Ulysses during the many years of its development phase. We wish to thank Fred Ipavich, Fritz Gliem, and Wolfgang Rieck for developing and implementing the on-board data reduction algorithms which enabled high data recovery despite the limited telemetry. Of particular benefit have been the many illuminating discussions with Len Fisk, Nathan Schwadron and Thomas Zurbuchen concerning evolution of the distribution functions of pickup ions, with Daniel Rucinski regarding ionization rates of interstellar atoms in the heliosphere and with Pricilla Frisch and Vlad Izmodenov on abundances in the LIC. We thank Christine Gloeckler for her help with data reduction. This work was supported in part by NASA/JPL contract 955460 (GG) and by the International Space Science Institute and the Swiss National Science Foundation (JG).

\section{References}

Anders, E. and Grevesse, N.: 1988, Geochim. Cosmochim. Acta 53, 197.

Axford, W. I.: 1972, in C. P. Sonett, P. J. Coleman, Jr., and J. M. Wilcox (eds.), Proc. Solar Wind Conf., Asilomar, California, 1971, NASA SP-308, p. 609.

Banks, P. M.: 1971, J. Geophys. Res. 76, 4341. 
Balogh, A., Gonzalez-Esparza, J. A., Forsyth, R. J., Burton, M. E., Goldstein, B. E., Smith, E. J., and Bame, S. J.: 1995, Space Sci. Rev. 72, 171.

Baranov, V. B. and Malama, Y. G.: 1995, J. Geophys. Res. 100, 755.

Blum, P. W. and Fahr, H. J.: 1970, Astron. Astrophys. 4, 280.

Brandt, J. C., A'Hearn, M. F., Randall, C. E., Schleicher, D. G., Shoemaker, E. M., and Stewart, A. I. F.: 1996, Earth, Moon and Planets 72, 243.

Fahr, H. J., Ripken, H. W., and Lay, G.: 1981, Astron. Astrophys. 102, 359.

Fisk, L. A.: 1996, Space Sci. Rev. 78, 129.

Fisk, L. A., Schwadron, N. A., and Gloeckler, G.: 1997, Geophys. Res. Letters 24, 93.

Fränz, M., Keppler, E., Krupp, N., Rouss, M. K., and Blake, J. B.: 1995, Space Sci. Rev. 72, 339.

Frisch, P. C.: 1996 ISSI Workshop, Space Sci. Revs. 78, 213.

Frisch, P. C.: 1998, Space Sci. Rev. 86, 107.

Geiss, J. and Witte, M.: 1996, Space Sci. Rev. 78, 229.

Geiss, J., Gloeckler, G., Balsiger H., Fisk, L. A., Galvin, A. B., Gliem, F., Hamilton, D. C., Ipavich, F. M., Livi, S., Mall, U., Ogilvie, K. W., von Steiger, R., and Wilken, B.: 1992, Science 257, 1535.

Geiss, J., Gloeckler, G., Mall, U., von Steiger, R., Galvin, A. B., and Ogilvie, K. W.: 1994a, Astron. Astrophys. 282, 924.

Geiss, J., Gloeckler, G., and Mall, U.: 1994b, Astron. Astrophys. 282, 933.

Geiss, J., Gloeckler, G., Fisk, L. A., and von Steiger, R.: 1995, J. Geophys. Res. 100, 23373.

Geiss, J., Gloeckler, G., and von Steiger, R.: 1996, Space Sci. Rev. 78, 43.

Gloeckler, G.: 1996, Space Sci. Rev. 78, 335.

Gloeckler, G. and Geiss, J.: 1996, Nature 381, 210.

Gloeckler, G. and Geiss, J.: 1998, Space Sci. Rev. 84, 275.

Gloeckler, G., Hovestadt, D., and Fisk, L. A.: 1979, Astrophys. J. 230, L191.

Gloeckler, G., Wilken, B., Stüdemann, W., Ipavich, F. M., Hovestadt, D., Hamilton, D. C., and Kremser, G.: 1985, Geophys. Res. Letters 12, 325.

Gloeckler, G., Geiss, J., Balsiger, H., Bedini, P., Cain, J. C., Fischer, J., Fisk, L. A., Galvin, A. B., Gliem, F., Hamilton, D. C., Hollweg, J. V., Ipavich, F. M., Joss, R., Livi, S., Lundgren, R., Mall, U., McKenzie, J. F., Ogilvie, K. W., Ottens, F., Rieck, W., Tums, E. O., von Steiger, R., Weiss, W., and Wilken, B.: 1992, Astron. Astrophys. Suppl. Ser. 92, 267.

Gloeckler, G., Geiss, J., Balsiger, H., Fisk, L. A., Galvin, A. B., Ipavich, F. M., Ogilvie, K. W., von Steiger, R., and Wilken, B.: 1993, Science 261, 70.

Gloeckler, G., Geiss, J., Roelof, E. C., Fisk, L. A., Ipavich, F. M., Ogilvie, K. W., Lanzerotti, L. J., von Steiger, R., and Wilken, B.: 1994, J. Geophys. Res. 99, 17637.

Gloeckler, G., Schwadron, N. A., Fisk, L. A., and Geiss, J.: 1995, Geophys. Res. Letters 22, 2665.

Gloeckler, G., Fisk, L. A., and Geiss, J: 1997, Nature 386, 374.

Grün, E., Zook, H. A., Fechtig, H., and Giese, R. H.: 1985, in R. H. Giese and P. Lamy (eds.), Mass Input Into and Output from the Meteoritic Complex in Properties and Interactions of Interplanetary Dust, D. Reidel Publ. Co., Dordrecht, Holland, pp. 411-430.

Gruntman, M.: 1996, J. Geophys. Res. 101, 15555.

Grünwaldt, H., Neugebauer, M., Hilchenbach, M., Bochsler, P., Hovestadt, D., Bürgi, A., Ipavich, F. M., Reiche, K.-U., Axford, W. I., Balsiger, H., Galvin, A. B., Geiss, J., Gliem, F., Gloeckler, G., Hsieh, K. C., Kallenbach, R., Klecker, B., Livi, S., Lee, M. A., Managadze, G. G., Marsch, E., Möbius, E., Scholer, M., Verigin, M. I., Wilken, B., and Wurz, P.: 1997, Geophys. Res. Letters 24, 1163 .

Holzer, T. E.: 1989, Ann. Rev. Astron. Astrophys. 27, 199.

Hovestadt, D., Klecker, B., Gloeckler, G., Ipavich, F. M., and Scholer, M: 1984, Astrophys. J. 282, L39.

Lallement, R.: 1996, Space Sci. Rev. 78, 361. 
Linsky, J. L., Diplas, A., Wood, B. E., Brown, A., Ayres, T. R., and Savage, B. D.: 1995, Astrophys. J. 451, 335 .

McNutt, R. L., Belcher, J. W., and Bridge, H. S.: 1981, J. Geophys. Res. 86, 8319.

Möbius, E.: 1996, Space Sci. Rev. 78, 375.

Möbius, E., Hovestadt, D., Klecker, B., Scholer, M., Gloeckler, G., and Ipavich, F. M.: 1985, Nature 318, 426.

Neugebauer, M., Lazarus, A. J., Altwegg, K., Balsiger, H., Schwenn, R., Shelley, E. G., and Ungstrup, E.: 1987, Astron. Astrophys. 187, 21.

Ogilvie, K. W., Gloeckler, G., and Geiss, J.: 1995, Astron. Astrophys. 299, 925.

Quemerais, E., Bertaux, J.-L., Sandel, B. R., and Lallement, R.: 1996, Astron. Astrophys. 290, 961.

Reames, D. V., Richardson, I. G., and Barbier, L. M.: 1991, Astrophys. J. 382, L43.

Rucinski, D., Cummings, A. C., Gloeckler, G., Lazarus, A. J., Möbius, E. and Witte, M.: 1996, Space Sci. Rev. 78, 361.

Rucinski, D., Bzowski, M., and Fahr, H.-J.: 1998, Astron. Astrophys., in press.

Schwadron, N. A., Fisk, L. A., and Gloeckler, G.: 1996, Geophys. Res. Letters 23, 2871.

Thomas, G. E.: 1978, Ann. Rev. Earth Planetary Sci. 6, 173.

Vasyliunas, V. M. and Siscoe, G. L.: 1976, J. Geophys. Res. 81, 1247.

Wimmer-Schweingruber, R. F., von Steiger, R., and Paerli, R.: 1997, J. Geophys. Res. 102, 17407.

Witte, M., Banaszkiewicz, M., and Rosenbauer, H.: 1996, Space Sci. Rev. 78, 289.

Witte, M., Rosenbauer, H., Banaszkiewicz, M., and Fahr, H. J.: 1993, Adv. Space Res. 13 (6), 121.

Young, D. T., Geiss, J., Balsiger, H., Eberhardt, P., Ghielmetti, A., and Rosenbauer, H.: 1977, Geophys. Res. Letters 4, 561. 